\title{
Consumption and Portfolio Choice under Internal Multiplicative Habit Formation*
}

\author{
Servaas van Bilsen \\ Dept. of Quantitative Economics \\ University of Amsterdam \\ and NETSPAR
}

\author{
A. Lans Bovenberg \\ Department of Economics \\ Tilburg University \\ CentER and NETSPAR
}

\author{
Roger J. A. Laeven ${ }^{\dagger}$ \\ Dept. of Quantitative Economics \\ University of Amsterdam \\ EURANDOM and CentER
}

Thursday $21^{\text {st }}$ March, 2019

\begin{abstract}
This paper explores the optimal consumption and investment behavior of an individual who derives utility from the ratio between his consumption and an endogenous habit. We obtain closed-form policies under general utility functionals and stochastic investment opportunities, by developing a non-trivial linearization to the budget constraint. This enables us to explicitly characterize how habit formation affects the marginal propensity to consume and optimal stock-bond investments. We also show that in a setting which combines habit formation with Epstein-Zin utility, consumption no longer grows at unrealistically high rates at high ages and investments in risky assets decrease.
\end{abstract}

JEL classification: D15, D81, G11, G13.

Keywords: Internal Habit Formation, Epstein-Zin Utility, Pathwise Approximation Technique, Return Smoothing, Life-Cycle Investment.

*We are very grateful to the Editor and an anonymous referee for comments and suggestions that have significantly improved the paper. We are also grateful to Yacine Aït-Sahalia, Markus Fels (discussant), Glenn Harrison, Gur Huberman, Frank de Jong, Torsten Kleinow (discussant), Olivia Mitchell, Theo Nijman, Antoon Pelsser (discussant), Hato Schmeiser (discussant), and to seminar and conference participants at Oxford, CEPAR in Sydney, Tilburg, Tinbergen Institute, Vienna, the University of Amsterdam, the University of Liverpool, the University of Pennsylvania (Wharton School), the Australasian Finance and Banking Conference, the CEAR/MRIC Behavioral Insurance Workshop, the European Group of Risk and Insurance Economists (EGRIE) Annual Meeting, the Netspar International Pension Workshop, the Quantitative Methods in Finance Conference, and the Winter School on Mathematical Finance for their helpful comments and suggestions. An earlier version of this paper was circulated under the title "How to invest and spend wealth in retirement? A utilitybased analysis." This research was supported in part by the Netherlands Organization for Scientific Research (NWO) under grant NWO VIDI 2009 (van Bilsen, Laeven) and by the European Commission under the seventh framework program (EU-MOPACT; Van Bilsen, Bovenberg). Conflicts of interest: none. Email addresses: S.vanBilsen@uva.nl, A.L.Bovenberg@uvt.nl, and R.J.A.Laeven@uva.nl.

$\dagger$ Corresponding author. Mailing Address: PO Box 15867, 1001 NJ Amsterdam, The Netherlands. Phone: +31 (0) 205254219 . 


\section{Introduction}

THE INTERNAL HABIT FORMATION LITERATURE, in which individuals draw utility from consumption relative to an endogenous habit, can be divided along two main model specifications that have been widely used in economics and finance: additive habits 1 (Constantinides (1990) ) and multiplicative habits Abel (1990) . $^{2}$ While both models are appealing from a prescriptive and a descriptive point of view, the latter model specification, also referred to as the ratio internal habit model, is advocated in particular by Carroll $(2000)$ and Fuhrer (2000). Just like the additive internal habit model, the ratio internal habit model can be rationalized (see, e.g., Crawford $(2010)$ and references therein) while, at the same time, it can account for the observed degree of excess smoothness in consumption. Contrary to the additive internal habit model, the ratio internal habit model does not require an artificial constraint on the individual's initial wealth position or on the habit dynamics to avoid negative infinite utility $\left.\right|^{3}$ Multiplicative internal habits play a central role in this paper.

A main ingredient of optimal consumption and portfolio choice problems is the temporal structure of preferences. The ratio internal habit model implies time-inseparability of preferences, but maintains a time additive structure in terms of relative consumption $]^{4}$ As is well-known, an additive structure where utility is additive over time and states of nature implies that the elasticity of intertemporal substitution (EIS) and risk aversion are linked. This is analytically convenient yet fairly restrictive. Therefore, we study multiplicative internal habits also under Epstein-Zin utility (Epstein and Zin (1989) $)^{5}$ This preference model decouples the EIS from risk aversion and has been widely used in the consumption and portfolio choice literature. ${ }^{6}$

\footnotetext{
${ }^{1}$ Additive habits are also referred to as subtractive habits, linear habits, or the difference habit model.

${ }^{2}$ Some authors assume that habits are external rather than internal; see, e.g., Abel (1990)'s catchingup-with-the-Joneses specification, Campbell and Cochrane (1999), and Chan and Kogan (2002).

${ }^{3}$ See, e.g., Carroll (2000) and Munk (2008) for a discussion of this point.

${ }^{4}$ Henceforth, we define relative consumption as the ratio between consumption and the habit level.

${ }^{5}$ Strictly speaking, we consider stochastic differential utility (SDU), which arises as a continuous-time limit of Epstein-Zin utility; see Duffie and Epstein (1992). See also Kraft and Seifried (2014) who show that Epstein-Zin utility converges to SDU.

${ }^{6}$ See, e.g., Campbell and Viceira (1999), Campbell, Cocco, Gomes, Maenhout, and Viceira (2001), Chacko and Viceira (2005), and Bhamra and Uppal (2006).
} 
In this paper we develop a closed-form approach to solve consumption and portfolio choice problems involving multiplicative internal habits. To analyze how multiplicative internal habit formation affects the conventional wisdom on optimal consumption and investment dynamics, we apply our general approach to three important cases: a base case with multiplicative internal habits, additive utility in terms of relative consumption, and constant investment opportunities; an extension of the base case with stochastic investment opportunities involving stochastic interest rates; and a case which combines multiplicative internal habits with Epstein-Zin utility.

In a nutshell, our approach consists of first applying a change of variables, redefining consumption in relative terms, and next pursuing a suitable pathwise linearization of the static budget constraint around the endogenous habit level 7 Our approximation approach transforms consumption and portfolio choice problems with multiplicative internal habits into approximate consumption and portfolio choice problems without habits. This enables us to obtain closed-form approximate solutions to a variety of consumption and portfolio choice problems with multiplicative internal habit formation under general utility functionals and stochastic investment opportunities.

We can summarize our three main findings as follows. First, we characterize in explicit closed-form how a habit-forming individual in the base-line model adjusts both his current consumption level and future growth rates of consumption after a stock return shock. While consumption is well-known to be excessively smooth under the ratio internal habit model, an explicit closed-form characterization of the shock absorbing mechanism is new. We show that the features of the marginal propensity to consume (i.e., shock absorbing mechanism) and investment strategy are determined by two factors: the degree of relative risk aversion and the strength of habit persistence. These factors not only have clear economic interpretations themselves but also induce clearly interpretable implications for the optimal consumption and portfolio decisions: the degree of relative risk aversion determines how large the impact of a stock return

\footnotetext{
${ }^{7}$ Linearization of the static budget constraint is not uncommon in the economics literature; see, in a different context, e.g., Campbell and Mankiw (1991), and Fuhrer (2000).
} 
shock is on the individual's current consumption level 8 and the strength of habit persistence determines the horizon-dependent impact of a stock return shock on future growth rates of consumption.$^{910}$ By contrast, under conventional constant relative risk aversion (CRRA) utility, which has become a main benchmark since Merton (1969), the impact of a stock return shock on consumption is uniformly distributed over time: it does not depend on the time distance between the occurrence of the shock and the date of consumption. We also find that an increase in habit persistence leads to a riskier investment strategy while leaving the year-on-year consumption volatility unaffected.11 As a result, current consumption of a habit-forming individual is less volatile than his underlying investment portfolio. Furthermore, we show that a habit-forming individual implements a life-cycle investment strategy that is nearly independent of the state of the economy (especially at high ages) and depends only on age ${ }^{12}$ Contrary to under conventional CRRA utility, we do not need human capital to justify a life-cycle investment strategy ${ }^{13}$

Second, in an extension of our base-line model that allows for stochastic interest rates and stock-bond investments, we find that the (interest rate) duration of the optimal hedging bond portfolio is hump shaped over the life cycle, which contradicts the conventional wisdom that the duration of the optimal hedging bond portfolio is decreasing with age. Two counteracting forces determine the life-cycle pattern of the

\footnotetext{
${ }^{8}$ In particular, we show that a stock return shock has a smaller impact on the current consumption level of a highly risk-averse individual than on that of a weakly risk-averse individual.

${ }^{9}$ We find that the more persistent the habit level is, the larger the impact of a stock return shock on future growth rates of consumption will be.

${ }^{10}$ We argue that the optimal policies provide a preference-based justification for the existence of annuity products in which surpluses earned in good years support benefit payouts in bad years. Such annuity products have been analyzed by e.g., Guillén, Jørgensen, and Nielsen (2006), Jørgensen and Linnemann (2012), Guillén, Nielsen, Pérez-Marín, and Petersen (2013), Maurer, Rogalla, and Siegelin (2013a), Linnemann, Bruhn, and Steffensen (2014), and Maurer, Mitchell, Rogalla, and Siegelin (2016).

${ }^{11}$ This finding stands in sharp contrast to standard unit-linked insurance products and traditional drawdown strategies in which a more aggressive portfolio strategy directly translates into a higher year-on-year consumption volatility. See, e.g., Dus, Maurer, and Mitchell (2005), Horneff, Maurer, Mitchell, and Dus (2008), and Maurer, Mitchell, Rogalla, and Kartashov (2013b) for a description of these products.

${ }^{12}$ More specifically, the individual in our base-line model lowers the share of his portfolio invested in the risky stock as he becomes older. Indeed, the available time to adjust current and future consumption levels in response to a stock return shock declines with age.

${ }^{13}$ For the classical implications of human capital on the optimal portfolio allocation, see Bodie, Merton, and Samuelson (1992) and Cocco, Gomes, and Maenhout (2005).
} 
duration of the optimal hedging bond portfolio. On the one hand, the impact of an interest rate shock on the price of future consumption is larger the younger the individual is. This effect causes the duration of the optimal hedging bond portfolio to decrease with age and is familiar from Brennan and Xia (2002) (see also Merton (2014)). On the other hand, we find a new effect that causes the duration of the optimal hedging bond portfolio to increase with age. We can explain this effect by the fact that a habit-forming individual is less willing to substitute consumption over time as he grows older. Intuitively, as the individual grows older, the duration of remaining lifetime consumption declines, and hence the current habit level determines to a greater extent future consumption levels ${ }^{14}$

A general feature of many habit formation models (including our base-line model) is that median consumption grows at unrealistically high rates (especially at high ages) except when the time discount rate is excessive. We therefore also analyze a model that combines Epstein-Zin utility with multiplicative internal habits 15 Our third main finding is, then, that in this setting that decouples the EIS from risk aversion, habit formation does not necessarily lead to unrealistically high unconditional median growth rates of consumption at the end of life, even when the time discount rate is moderate. Furthermore, wealth accumulation is substantially lower under this extended model than under the base-line model. Hence, an individual whose preferences combine multiplicative internal habit formation with Epstein-Zin utility invests less wealth in the stock market compared to an individual without Epstein-Zin utility.

The endogenous nature of the habit in internal habit formation models substantially complicates the analysis of optimal consumption and portfolio policies and asset pricing problems. In important work, Schroder and Skiadas (2002) show how to transform models with additive internal habits into models without habit formation, enabling closed-form solutions to a wide range of asset pricing problems involving additive internal habits. ${ }^{16}$

\footnotetext{
${ }^{14}$ The second effect may explain why not many young individuals include long-term bonds in their investment portfolios; see Morningstar (2017) for the investment behavior of long-term investors.

${ }^{15}$ The closest to the current paper in this respect is Schroder and Skiadas (1999) who analytically studied Epstein-Zin utility but did not consider multiplicative internal habits.

${ }^{16}$ See, e.g., Van Bilsen, Laeven, and Nijman (2017) who employ Schroder and Skiadas (2002) to explicitly derive the optimal consumption and portfolio policies under loss aversion and endogenous
} 
Conversely, their approach allows to translate solutions to familiar consumption and portfolio choice problems under general utility functionals into solutions to corresponding problems exhibiting additive internal habit formation. So far, however, internal habit formation models with multiplicative habits cannot be solved analytically. Thus, analysis of the appealing ratio internal habit model necessarily resorted to numerical methods to obtain solutions, impeding their applicability.

We obtain closed-form solutions to consumption and portfolio choice problems featuring multiplicative internal habits based on developing a pathwise approximation to the budget constraint. Our numerical results show that the approximation error, when measured in terms of the relative decline in certainty equivalent consumption, is typically less than 1\%, and that the explicit optimal policies to the approximate problems closely mimic the numerically evaluated optimal policies to the original problems. Having closed-form solutions has three key advantages: they reveal the roles played by the various model parameters, they are readily amenable to comparative statics analysis, and they facilitate the implementation of the optimal consumption and investment policies.

We note that the problem of optimal consumption and portfolio choice over the life cycle has intrigued many authors since the seminal work of Mossin (1968), Merton (1969, 1971), and Samuelson (1969). Their work has been extended along many dimensions ${ }^{17}$ Many life-cycle consumption and portfolio choice papers assume a standard preference model; that is, decision makers' preferences are described by time-additive CRRA utility or Epstein-Zin Constant EIS-CRRA utility. While these standard preference models satisfy a set of normatively compelling axioms, their ability to describe how people actually make decisions under risk is known to be limited. Furthermore, their predictions fail to explain well-documented facts about actual consumption and portfolio behavior such as the excess smoothness of consumption. The updating of the reference level - two key features of prospect theory (Tversky and Kahneman (1992)).

${ }^{17}$ For instance, to accommodate time-varying investment opportunities (see, e.g., Campbell et al. (2001), Wachter (2002), Chacko and Viceira (2005), Liu (2007), and Laeven and Stadje (2014)); uncertain labor income (see, e.g., Viceira (2001), Cocco et al. (2005), and Gomes and Michaelides (2005)); housing costs (see, e.g., Cocco (2005), and Yao and Zhang (2005)); and unexpected health expenditures (see, e.g., Edwards (2008)). 
shortcomings of standard preference models have inspired many researchers to develop alternative theories for decision-making under risk ${ }^{18}$ including habit formation.

Several authors have explored the implications of these alternative preference theories for optimal investment decisions or intertemporal consumption behavior.19 Most relevant to our base-line model are Detemple and Zapatero (1991, 1992), Schroder and Skiadas (2002), Bodie, Detemple, Otruba, and Walter (2004) and Munk (2008) who analyze the optimal consumption and investment behavior of an individual who derives utility from the difference between consumption and an internal habit level, rather than some ratio of these as we do. Contrary to under the ratio habit model, the optimal consumption choice implied by the difference habit model exceeds the habit level in each economic scenario. This so-called addictive behavior of consumption is criticized theoretically e.g., by Chapman (1998) and Carroll (2000), and arguably at odds with empirical evidence. ${ }^{20}$ Finally, the ratio habit model has been employed in other papers to analyze monetary policy (Fuhrer $(2000))$, asset prices with an external habit Abel (1999), Chan and Kogan (2002) and Gómez, Priestley, and Zapatero (2009)) and an internal habit (Smith and Zhang (2007)), macroeconomic growth (Carroll, Overland, and Weil (1997), Carroll, Overland, and Weil (2000) and Carroll (2000)), and portfolio choice with uninsurable labor income risk (Gomes and Michaelides $(2003)$ ).

\section{Model}

\subsection{Asset Prices, Pricing Kernel and Budget Constraint}

Denote by $T>0$ a fixed terminal time. We represent the randomness in the economy by a filtered probability space $(\Omega, \mathcal{F}, \mathbb{F}, \mathbb{P})$ on which we define a standard $N$-dimensional

\footnotetext{
${ }^{18}$ Among the most notable alternatives are prospect theory (Kahneman and Tversky $(1979)$, and Tversky and Kahneman (1992)), regret theory (Loomes and Sugden (1982), Bell (1982,|1983), Sugden (1993), and Quiggin (1994)), disappointment (aversion) theory (Bell (1985), Loomes and Sugden (1986), and Gul (1991)), and habit formation (Abel (1990), Constantinides (1990) and Sundaresan (1989)).

${ }^{19}$ See, e.g., Bowman, Minehart, and Rabin (1999), Berkelaar, Kouwenberg, and Post (2004), Ang, Bekaert, and Lui (2005), Muermann, Mitchell, and Volkman (2006), Guasoni, Huberman, and Ren (2015), Pagel (2017) and Van Bilsen et al. (2017).

${ }^{20}$ For instance, Crossley, Low, and O'Dea (2013) show that consumption levels declined significantly during recent recessions, contradicting the addictive property of consumption.
} 
Brownian motion $\left\{W_{t}\right\}_{0 \leq t \leq T}$. The filtration $\mathbb{F}=\left\{\mathcal{F}_{t}\right\}_{0 \leq t \leq T}$ is the augmentation under $\mathbb{P}$ of the natural filtration generated by $\left\{W_{t}\right\}_{0 \leq t \leq T}$. Throughout, (in)equalities between random variables hold $\mathbb{P}$-almost surely.

We consider a financial market consisting of a risk-free asset and $N$ risky assets. Trading takes place continuously over $[0, T]$. The price of the risk-free asset, $B_{t}$, satisfies

$$
\frac{\mathrm{d} B_{t}}{B_{t}}=r_{t} \mathrm{~d} t, \quad B_{0}=1
$$

We assume that the scalar-valued risk-free rate process, $\left\{r_{t}\right\}_{0 \leq t \leq T}$, is $\mathcal{F}_{t}$-progressively measurable and satisfies $\int_{0}^{T}\left|r_{t}\right| \mathrm{d} t<\infty$. The $N$-dimensional vector of risky asset prices, $S_{t}$, obeys the following stochastic differential equation:

$$
\frac{\mathrm{d} S_{t}}{S_{t}}=\mu_{t} \mathrm{~d} t+\sigma_{t} \mathrm{~d} W_{t}, \quad S_{0}=\mathbf{1}_{N}
$$

Here, $\mathbf{1}_{N}$ represents an $N$-dimensional vector consisting of all ones. We assume that the $N$-dimensional mean rate of return process, $\left\{\mu_{t}\right\}_{0 \leq t \leq T}$, and the $(N \times N)$-matrix-valued volatility process, $\left\{\sigma_{t}\right\}_{0 \leq t \leq T}$, are $\mathcal{F}_{t}$-progressively measurable and satisfy $\int_{0}^{T}\left\|\mu_{t}\right\| \mathrm{d} t<$ $\infty$ and $\sum_{i=1}^{N} \sum_{j=1}^{N} \int_{0}^{T}\left(\sigma_{t}\right)_{i j}^{2} \mathrm{~d} t<\infty$, respectively. We impose the following additional condition on $\sigma_{t}$. For some $\epsilon>0$,

$$
\zeta^{\top} \sigma_{t} \sigma_{t}^{\top} \zeta \geq \epsilon\|\zeta\|^{2}, \quad \text { for all } \zeta \in \mathbb{R}^{N}
$$

with $\top$ denoting the transpose sign. Condition (3) implies that $\sigma_{t}$ is invertible.

The $\mathcal{F}_{t}$-progressively measurable market price of risk process, $\left\{\lambda_{t}\right\}_{0 \leq t \leq T}$, satisfies

$$
\sigma_{t} \lambda_{t}=\mu_{t}-r_{t} \mathbf{1}_{N}
$$

The unique positive-valued state price density process, $\left\{M_{t}\right\}_{0 \leq t \leq T}$, is given by (see, e.g., Karatzas and Shreve (1998)):

$$
M_{t}=\exp \left\{-\int_{0}^{t} r_{s} \mathrm{~d} s-\int_{0}^{t} \lambda_{s}^{\top} \mathrm{d} W_{s}-\frac{1}{2} \int_{0}^{t}\left\|\lambda_{s}\right\|^{2} \mathrm{~d} s\right\}
$$


The economy consists of an individual endowed with initial wealth $A_{0} \geq 0$. This individual chooses an $\mathcal{F}_{t}$-progressively measurable $N$-dimensional portfolio process $\left\{\pi_{t}\right\}_{0 \leq t \leq T}$ (representing the amounts invested in the $N$ risky assets) and an $\mathcal{F}_{t}$-progressively measurable consumption process $\left\{c_{t}\right\}_{0 \leq t \leq T}$ so as to maximize lifetime utility. We impose the following conditions on the portfolio and consumption processes:

$$
\int_{0}^{T} \pi_{t}^{\top} \sigma_{t} \sigma_{t}^{\top} \pi_{t} \mathrm{~d} t<\infty, \quad \int_{0}^{T}\left|\pi_{t}\left(\mu_{t}-r_{t} \mathbf{1}_{N}\right)\right| \mathrm{d} t<\infty, \quad \mathbb{E}\left[\int_{0}^{T}\left|c_{t}\right|^{r} \mathrm{~d} t\right]<\infty \forall r \in \mathbb{R}
$$

The wealth process, $\left\{A_{t}\right\}_{0 \leq t \leq T}$, satisfies the following dynamic budget constraint:

$$
\mathrm{d} A_{t}=\left(r_{t} A_{t}+\pi_{t}^{\top} \sigma_{t} \lambda_{t}-c_{t}\right) \mathrm{d} t+\pi_{t}^{\top} \sigma_{t} \mathrm{~d} W_{t}, \quad A_{0} \geq 0 \text { given }
$$

A consumption-portfolio pair $\left\{c_{t}, \pi_{t}\right\}_{0 \leq t \leq T}$ is admissible if the wealth process is positive.

\section{$2.2 \quad$ Habit Level}

Denote by $h_{t}$ the individual's habit level at time $t$. Following Kozicki and Tinsley (2002) and Corrado and Holly (2011), we assume that the log habit level $\log h_{t}$ satisfies the following dynamic equation: ${ }^{21}$

$$
\mathrm{d} \log h_{t}=\left(\beta \log c_{t}-\alpha \log h_{t}\right) \mathrm{d} t, \quad \log h_{0}=0 .
$$

We normalize the initial $\log$ habit $\log h_{0}$ to zero, i.e., $h_{0}$ equals unity. The preference parameter $\alpha \geq 0$ represents the rate at which the log habit level exponentially depreciates. When $\alpha$ is small, the log habit level exhibits a high degree of memory. The preference parameter $\beta \geq 0$ models the relative importance between the initial habit level and the individual's past consumption choices. When $\beta$ is large, the individual's past consumption choices are relatively important. We assume that $\alpha \geq \beta$. This parameter restriction prevents the individual's habit level from growing exponentially over time; see Eqn. (13)

\footnotetext{
${ }^{21}$ The $\log$ habit level $\log h_{t}$ is additive, i.e., linear, in past levels of log consumption. Corrado and Holly (2011) show that for the ratio internal habit model, the habit specification (8) is more desirable than an arithmetic habit specification in which the habit level $h_{t}$ itself is additive in past levels of consumption.
} 
below. In the special case where $\beta=0$, the habit level is exogenously given.

\subsection{Dynamic Optimization Problem}

Let $U(c / h) \in \mathbb{R} \cup\{-\infty\}$ be the individual's lifetime utility derived from the process $c / h=\left\{c_{t} / h_{t}\right\}_{0 \leq t \leq T}$ representing the ratio between consumption and the habit level. We

place no restrictions on $U$. The individual now faces the following dynamic optimization problem over admissible consumption-portfolio pairs $\left\{c_{t}, \pi_{t}\right\}_{0 \leq t \leq T}$ :

$$
\begin{array}{rl}
\max _{c_{t}, \pi_{t}: 0 \leq t \leq T} & U\left(\frac{c}{h}\right) \\
\text { s.t. } & \mathrm{d} A_{t}=\left(r_{t} A_{t}+\pi_{t}^{\top} \sigma_{t} \lambda_{t}-c_{t}\right) \mathrm{d} t+\pi_{t}^{\top} \sigma_{t} \mathrm{~d} W_{t}, \quad A_{0} \geq 0 \text { given, } \\
& \mathrm{d} \log h_{t}=\left(\beta \log c_{t}-\alpha \log h_{t}\right) \mathrm{d} t .
\end{array}
$$

Section 3 presents a solution technique for analytically solving (9) based on developing a pathwise linearization to the individual's budget constraint.

\section{Solution Method}

\subsection{An Equivalent Problem}

We can, by virtue of the martingale approach (Pliska (1986), Karatzas, Lehoczky, and Shreve (1987), and Cox and Huang (1989, 1991)), transform the individual's dynamic optimization problem (9) into the following equivalent static variational problem:

$$
\begin{array}{rl}
\max _{c_{t}: 0 \leq t \leq T} & U\left(\frac{c}{h}\right) \\
\text { s.t. } & \mathbb{E}\left[\int_{0}^{T} M_{t} c_{t} \mathrm{~d} t\right] \leq A_{0}, \\
& \mathrm{~d} \log h_{t}=\left(\beta \log c_{t}-\alpha \log h_{t}\right) \mathrm{d} t,
\end{array}
$$

where $M_{t}$ is given by (5).

After the optimal consumption choice $c_{t}^{\text {opt }}$ has been determined, one can determine the optimal portfolio choice $\pi_{t}^{\mathrm{opt}}$ using hedging arguments. 


\subsection{A Change of Variable Transformation}

Denote by $\widehat{c}_{t}$ the ratio between the consumption choice and the habit level; that is,

$$
\widehat{c}_{t}=\frac{c_{t}}{h_{t}}
$$

We can express the dynamics of the log habit level in terms of the individual's log relative consumption choice $\log \widehat{c}_{t}=\log \left(c_{t} / h_{t}\right)$ as follows:

$$
\mathrm{d} \log h_{t}=\left(\beta \log \widehat{c}_{t}-[\alpha-\beta] \log h_{t}\right) \mathrm{d} t
$$

Hence, the individual's log habit level $\log h_{t}$ is explicitly given by

$$
\log h_{t}=\beta \int_{0}^{t} \exp \{-(\alpha-\beta)(t-s)\} \log \widehat{c}_{s} \mathrm{~d} s
$$

Eqn. 13 shows that, as a result of the parameter restriction $\alpha \geq \beta$, the individual's habit level is prevented from growing exponentially over time.

We can thus rewrite the individual's static optimization problem 10 in terms of $\widehat{c}_{t}=c_{t} / h_{t}$ yielding the following equivalent problem:

$$
\begin{array}{rl}
\max _{\widehat{c}_{t}: 0 \leq t \leq T} & U(\widehat{c}) \\
\text { s.t. } & \mathbb{E}\left[\int_{0}^{T} M_{t} h_{t} \widehat{c}_{t} \mathrm{~d} t\right] \leq A_{0}, \\
& \mathrm{~d} \log h_{t}=\left(\beta \log \widehat{c}_{t}-[\alpha-\beta] \log h_{t}\right) \mathrm{d} t .
\end{array}
$$

We then obtain the optimal consumption choice $c_{t}^{\text {opt }}$ from the optimal relative consumption choice $\widehat{c}_{t}^{\text {opt }}$ as follows: ${ }^{22}$

$$
c_{t}^{\mathrm{opt}}=h_{t}^{\mathrm{opt}} \widehat{c}_{t}^{\mathrm{opt}}
$$

To solve the individual's static optimization problem (10), we can thus restrict ourselves to

\footnotetext{
${ }^{22}$ We determine $h_{t}^{\text {opt }}$ by substituting the optimal relative consumption choices $\widehat{c}_{s}^{\text {opt }}(s \leq t)$ into 13 .
} 
solving (14). In applications, it is still typically impossible to solve the static optimization problem (14) analytically. The reason for this is that the new static budget constraint in (14) depends non-linearly on the individual's relative consumption choices ${ }^{23}$ Section 3.3 develops a pathwise linearization for the new static budget constraint in (14). After applying this linearization, we are able to obtain analytical closed-form expressions for the individual's consumption and investment policies in a wide range of interesting cases.

\subsection{Linearization of the New Static Budget Constraint}

This section presents a linear approximation to the left-hand side of the new static budget constraint in (14) around the relative consumption trajectory $\left\{\widehat{c}_{t}\right\}_{0 \leq t \leq T}=1$. Consumption $c_{t}$ is thus approximated around the habit level $h_{t}$. The key insight here is that, because the habit level is determined endogenously by the individual's own past consumption choices, it tracks consumption. As a result, the habit level is a natural candidate around which to apply the approximation. This yields a "pathwise approximation'. Section 7 explores the approximation error induced by applying our pathwise approximation to the new static budget constraint in (14). The numerical results reveal that the approximation error is typically less than $1 \%$ in terms of relative decline in certainty equivalent consumption and that our closed-form approximated strategies closely mimic the genuinely optimal (but numerically evaluated) strategies.

Appendix A proves the following theorem.

Theorem 3.1. Consider an individual who aims to solve the optimization problem (10). This problem is equivalent to the following simpler problem up to a first-order approximation of the static budget constraint:

$$
\begin{array}{rl}
\max _{\widehat{c}_{t}: 0 \leq t \leq T} & U(\widehat{c}) \\
\text { s.t. } & \mathbb{E}\left[\int_{0}^{T} \widehat{M}_{t} \widehat{c}_{t} \mathrm{~d} t\right] \leq \widehat{A}_{0} .
\end{array}
$$

Here, $\widehat{M}_{t} \equiv M_{t}\left(1+\beta P_{t}\right)$ with $P_{t}$ denoting the price at time $t$ of a bond that pays the

\footnotetext{
${ }^{23}$ Indeed, substitution of the habit level $h_{t}$ (see (13)) into the budget constraint in (14) shows that the new static budget constraint in $(14)$ is non-linear in the individual's relative consumption choices.
} 
coupon process $\left\{e^{-(\alpha-\beta)(s-t)}\right\}_{s \geq t}$, i.e.,

$$
P_{t} \equiv \mathbb{E}_{t}\left[\int_{t}^{T} \frac{M_{s}}{M_{t}} e^{-(\alpha-\beta)(s-t)} \mathrm{d} s\right]
$$

The quantity $\widehat{A}_{0}$ denotes the individual's initial wealth associated with the approximate problem (16). We determine the individual's initial wealth $\widehat{A}_{0}$ such that the approximate optimal consumption strategy $\left\{c_{t}^{*}\right\}_{0 \leq t \leq T}=\left\{h_{t}^{*} \widehat{c}_{t}^{*}\right\}_{0 \leq t \leq T}$ is budget-feasible ${ }^{24}$

We emphasize that (16) is a standard complete markets problem. Indeed, the endogenous habit level $h_{t}$ does not appear in (16), thanks to the change of variable transformation and, crucially, our pathwise linearization of the static budget constraint. The relative consumption choice $\widehat{c}_{t}^{*}$ solving (16) is an approximation to the optimal relative consumption choice $\widehat{c}_{t}^{\text {opt }}$.

Remark 1. Using a suitable transformation, Schroder and Skiadas (2002) translate models with an internal habit and utility expressed as a function of the difference between consumption and a habit level into models without habit formation. Interestingly and quite surprisingly (to us), the transformed state price density process $\widehat{M}_{t}$ and hence the transformed problem $(16)$ are identical to the transformed counterparts in Schroder and Skiadas (2002), the difference being that $\widehat{c}_{t}$ represents surplus consumption $c_{t}-h_{t}$ in their framework while it represents relative consumption $c_{t} / h_{t}$ in our framework. In their setting, the original budget constraint is equivalent to the budget constraint in the transformed problem:

$$
\mathbb{E}\left[\int_{0}^{T} M_{t} c_{t} \mathrm{~d} t\right]=\mathbb{E}\left[\int_{0}^{T} \widehat{M}_{t}\left(c_{t}-h_{t}\right) \mathrm{d} t\right]+K_{1}
$$

while in our setting the new budget constraint first-order approximates the original budget constraint:

$$
\mathbb{E}\left[\int_{0}^{T} M_{t} c_{t} \mathrm{~d} t\right] \approx \mathbb{E}\left[\int_{0}^{T} \widehat{M}_{t} \frac{c_{t}}{h_{t}} \mathrm{~d} t\right]+K_{2}
$$

Here, $K_{1}$ and $K_{2}$ are constants that are irrelevant in determining the first-order optimality

\footnotetext{
${ }^{24}$ Here, $h_{t}^{*}$ denotes the habit level at time $t$ implied by substituting the approximate optimal past relative consumption choices $\widehat{c}_{s}^{*}(s \leq t)$ into 13 .
} 
conditions. Note also that the interpretation of the parameters $\alpha$ and $\beta$ is different in the two papers as we consider the dynamics of the log habit level $\log h_{t}$ while they consider the dynamics of the habit level $h_{t}$.

\section{Ratio Internal Habit Model}

This section assumes that lifetime utility is defined as follows:

$$
U\left(\frac{c}{h}\right)=\mathbb{E}\left[\int_{0}^{T} e^{-\delta t} \frac{1}{1-\gamma}\left(\frac{c_{t}}{h_{t}}\right)^{1-\gamma} \mathrm{d} t\right],
$$

with $\log h_{t}$ satisfying (8). Here, $\mathbb{E}$ is the unconditional expectation, $\delta \geq 0$ stands for the subjective rate of time preference, and $\gamma>0$ is the coefficient of relative risk aversion. Specification (20) corresponds to the habit formation model proposed by Abel (1990).

\subsection{Optimal Consumption Choice}

Theorem 4.1 presents the (approximate) optimal consumption choice $c_{t}^{*}$.

Theorem 4.1. Consider an individual with lifetime utility (20) and habit formation process (8) who solves the consumption and portfolio choice problem (16). Denote by $h_{t}^{*}$ the habit level at time $t$ implied by substituting the (approximate) optimal past relative consumption choices $\widehat{c}_{s}^{*}(s \leq t)$ into (13), and by $y$ the Lagrange multiplier associated with the static budget constraint in (16). Then the (approximate) optimal consumption choice $c_{t}^{*}$ is given by

$$
c_{t}^{*}=h_{t}^{*}\left(y e^{\delta t} \widehat{M}_{t}\right)^{-\frac{1}{\gamma}} .
$$

The Lagrange multiplier $y \geq 0$ is determined such that the individual's original budget constraint holds with equality.

Note that the expression of the approximate consumption strategy $\widehat{c}_{t}^{*}=c_{t}^{*} / h_{t}^{*}$ is well-known (see, e.g., Merton (1969)). It agrees exactly with $\widehat{c}_{t}^{\text {opt }}$ in case $\alpha=\beta=0$. 


\subsection{Sensitivity and Volatility of Future Consumption}

In the remainder of this section, we assume constant investment opportunities (i.e., $r_{t}=r, \mu_{t}=\mu, \sigma_{t}=\sigma$ and $\lambda_{t}=\lambda$ for all $t$ ) and only one risky stock. We set the risk-free interest rate $r$ at $1 \%$, the equity risk premium $e=\mu-r$ at $4 \%$, and the stock return volatility $\sigma$ at $20 \%$. These parameter values are the same as those used in Gomes, Kotlikoff, and Viceira (2008). The time preference rate $\delta$ is assumed to be equal to $3 \% .25$

Denote by $q_{t-s}$ the sensitivity of $\log$ consumption, $\log c_{t}^{*}$, to a past stock return shock, $\sigma \mathrm{d} W_{s}(s \leq t)$. We find the explicit closed-form expression (see Appendix A ${ }^{26}$

$$
q_{t-s}=\frac{\lambda}{\gamma \sigma} Q_{t-s}
$$

with

$$
Q_{t-s}=1+\frac{\beta}{\alpha-\beta}[1-\exp \{-(\alpha-\beta)(t-s)\}]
$$

The sensitivity $q_{t-s}$, dictating the optimal shock absorbing mechanism in analytical form, depends on the time distance between the date at which the stock return shock occurs (i.e., time $s$ ) and the date of consumption (i.e., time $t>s$ ). In particular, a closer inspection of 22 shows that $q_{\tau}$ increases with the horizon $\tau$ : a current stock return shock has a smaller impact on log consumption in the near future (i.e., small $\tau$ ) than on $\log$ consumption in the distant future (i.e., large $\tau$ ). In case the individual exhibits conventional constant relative risk aversion (CRRA) utility (henceforth referred to as a CRRA individual), which has become the main benchmark since Merton (1969), the sensitivity $q_{\tau}$ is independent of the horizon $\tau$.

Our utility framework thus provides a preference-based justification for the existence of annuity products in which current stock return shocks are not fully reflected into current annuity payouts. These products work as follows (see, e.g., Guillén et al. (2006), Linnemann et al. (2014), and Maurer et al. (2016)). In the case of a positive investment return, the annuity payout will go up by less than the realized return. The remaining

\footnotetext{
${ }^{25}$ Samwick $(1998)$ finds that median time preference rates for US households are between $3 \%$ and $4 \%$.

${ }^{26}$ We note that if $\alpha=\beta$, then (23) reduces to $Q_{t-s}=1+\beta(t-s)$.
} 
investment gains will be added to a reserve fund. In the case of a negative investment return, the annuity payout will be protected and will decrease by a lower percentage than the realized return. This 'payout protection' will be paid from the reserve fund. The just described mechanism results in an excessively smooth payout stream.

The individual's preference parameters $\gamma, \alpha$ and $\beta$ have clearly interpretable implications for the individual's optimal consumption choice. We find that a current stock return shock $\sigma \mathrm{d} W_{t}$ has a smaller impact on the current consumption level of a highly risk-averse individual (i.e., high $\gamma$ ) than on that of a weakly risk-averse individual (i.e., low $\gamma$ ). Indeed, a highly risky-averse individual is more risk averse to year-on-year fluctuations in current consumption than a weakly risk-averse individual. The coefficients $\beta$ and $\widehat{\alpha}=\alpha-\beta$, which measure the degree of habit persistence, determine the impact of a current stock return shock on the future growth rates of (median) consumption. If the individual's preferences exhibit a large degree of habit persistence (i.e., $\beta$ is large and $\widehat{\alpha}$ is close to zero), a current stock return shock will have a relatively large impact on future growth rates of consumption: the individual adjusts the future growth rates of consumption downwards (upwards) by a relatively large percentage after the occurrence of a negative (positive) stock return shock. Figure 1 illustrates the sensitivity $q_{\tau}$ as a function of the horizon $\tau$ for various parameter values.

Denote by $\Sigma_{t, \tau}$ the annualized volatility of future consumption $\log c_{t+\tau}^{*}$ at time $t$, i.e.,

$$
\Sigma_{t, \tau}:=\sqrt{\frac{\mathbb{V}_{t}\left[\log c_{t+\tau}^{*}\right]}{\tau}}
$$

Here, $\mathbb{V}_{t}$ denotes the variance conditional on the information available at time $t$. We find that the annualized volatility of the future consumption choice of an individual whose preferences exhibit internal habit formation depends on the horizon $\tau$. More specifically, the annualized volatility of $\log c_{t+\tau}^{*}$ is given in closed-form by

$$
\Sigma_{t, \tau}=\Sigma_{\tau}=\sqrt{\frac{\int_{0}^{\tau} q_{v}^{2} \mathrm{~d} v}{\tau}} \cdot \sigma
$$

Note that the annualized variance is proportional to the normalized integrated squared 


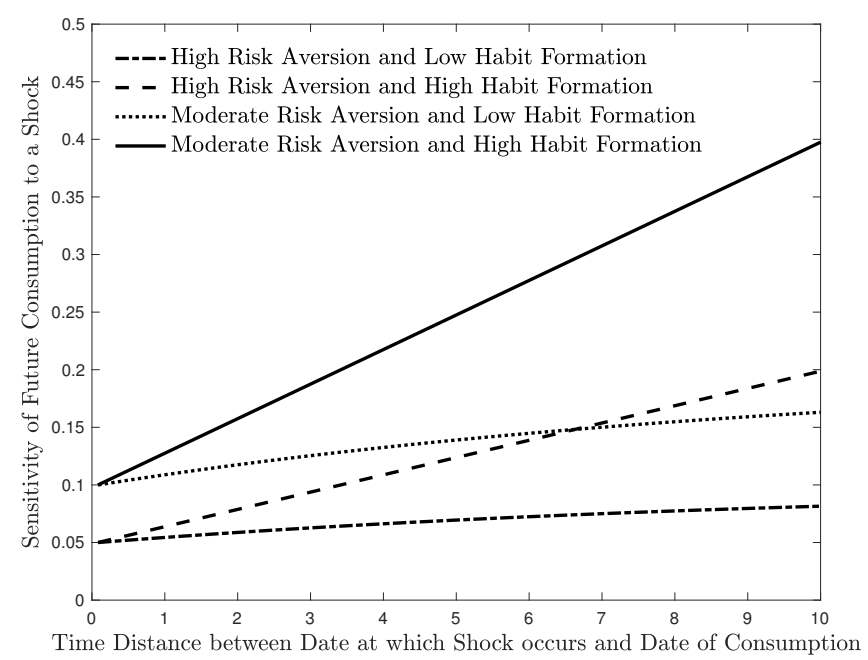

Figure 1: Sensitivity of future consumption. The figure illustrates the sensitivity of future consumption to a stock return shock (i.e., $q_{\tau}$ ) as a function of the horizon $\tau$ (i.e., the time distance between the date at which the stock return shock occurs and the date of consumption). The figure considers four different types of individuals: a highly risk-averse individual with a low degree of habit persistence (i.e., $\gamma=20, \alpha=0.2, \beta=0.1$ ); a highly risk-averse individual with a high degree of habit persistence (i.e., $\gamma=20, \alpha=\beta=0.3$ ); a moderately risk-averse individual with a low degree of habit persistence (i.e., $\gamma=10, \alpha=0.2, \beta=0.1$ ); and a moderately risk-averse individual with a high degree of habit persistence (i.e., $\gamma=10, \alpha=\beta=0.3$ ). In the case of a CRRA individual, the sensitivity of future consumption does not depend on the horizon $\tau$. We set both the market price of risk $\lambda$ and the stock return volatility $\sigma$ equal to 0.2 .

sensitivity $q_{\tau}$. Because $q_{\tau}$ increases with the horizon $\tau$, it follows that for an individual with habit preferences, the annualized volatility of consumption in the near future is smaller than the annualized volatility of consumption in the far future. Finally, we note that the annualized volatility of the future consumption choice of a CRRA individual does not depend on the horizon $\tau$ : consumption in the near future exhibits the same annualized volatility as consumption in the far future.

\subsection{Shock Absorbing Mechanism}

This section illustrates in more detail how the current and future consumption levels of an individual whose preferences exhibit internal habit formation respond to an unexpected stock return shock. We consider an individual who starts working at the age of 25 and passes away at the age of 85 . He invests and spends his accumulated wealth according to the ratio internal habit model $(20)$ with preference parameters $\gamma=10, \alpha=0.3$ and 
$\beta=0.3$. We also study our model findings for other degrees of habit persistence ${ }^{27}$ As we show below, our results remain qualitatively unchanged if we vary the degree of habit persistence. We assume that the individual adjusts consumption once a year ${ }^{28}$

We compare our findings to the optimal behavior of a CRRA individual. We assume that the CRRA individual invests $50 \%$ of his accumulated wealth in the stock market 29 His investment behavior roughly coincides with the investment behavior of a 58-yearold individual with habit preferences; for more details on the portfolio strategy of an individual with habit preferences, see Section 4.5 .

Figures 2(a) and (b) illustrate the impact of a $38 \% 30$ stock price decline in year one on current and future consumption choices. A CRRA individual fully translates a current stock return shock into his current consumption level. In this illustration, the current consumption level of a CRRA individual decreases by $19.35 \%$ after the stock price shock has been realized. The stock return shock does not affect the future growth rates of his consumption; see Figure 2(a) which shows that the shape of the median consumption path of a CRRA individual remains unaffected by a stock return shock.

An individual whose preferences exhibit internal habit formation does not fully translate a current stock return shock into his current consumption level. As a result, the relative decline in the current consumption level of an individual with habit preferences is typically smaller than the relative decline in the current consumption level of a CRRA individual. Indeed, his current consumption level drops by only $4.21 \%$ while the current consumption level of a CRRA individual drops by more than $19 \%$. The flip side of protecting current consumption is that the shape of the median consumption path cannot remain unchanged following a stock return shock; see Figure 2[(b) which shows that the individual adjusts the future growth rates of his median

\footnotetext{
${ }^{27}$ We note that the degrees of habit persistence we explore are considered reasonable by Fuhrer $(2000)$ and Gomes and Michaelides (2003).

${ }^{28}$ All figures and tables in this paper assume that the individual adjusts consumption only once a year. We note that this is not a restriction of our framework. We could also illustrate the case in which the individual adjusts consumption every month or every week.

${ }^{29}$ Assuming $\lambda=\sigma=0.2$, a portfolio weight of $50 \%$ implies a relative risk aversion coefficient of 2 in the Merton model (Merton (1969)).

${ }^{30}$ This number corresponds to the decline in the S\&P 500 index between January 1, 2008 and December $31,2008$.
} 
consumption downwards. A consequence of adjusting future growth rates is thus that the impact of a shock on median consumption is larger the longer the horizon is.

Figures 2(c) and (d) illustrate the impact of a $24 \% 31$ stock price increase in year two on current and future consumption choices. As in Figure 2|(a), the CRRA individual directly absorbs the current stock return shock into his current consumption level. The current stock return shock has a smaller impact on the current consumption level of an individual with habit preferences than on that of the CRRA individual. Indeed, an individual with habit preferences has a strong preference to protect current consumption. In fact, in this illustration, he only consumes slightly more than last year, because he has translated part of last year's (negative) stock return shock into consumption of this year. Furthermore, as a result of the current stock price increase, he adjusts the future growth rates of his median consumption upwards; see Figure 2| (d).

\subsection{Decomposition of the Consumption Dynamics}

We can decompose the dynamics of the individual's $\log$ consumption choice $\log c_{t}^{*}$ as follows (see Appendix A):

$$
\mathrm{d} \log c_{t}^{*}=g_{t} \mathrm{~d} t+p_{t} \mathrm{~d} t+\frac{\lambda}{\gamma \sigma} \sigma \mathrm{d} W_{t}
$$

Here,

$$
g_{t}=\frac{1}{\gamma}\left(\widehat{r}_{t}+\frac{1}{2} \lambda^{2}-\delta\right), \quad p_{t}=Q_{t}^{\prime} \log c_{0}^{*}+\frac{1}{\gamma} \int_{0}^{t} Q_{t-s}^{\prime} g_{s} \mathrm{~d} s+\frac{\lambda}{\gamma \sigma} \int_{0}^{t} Q_{t-s}^{\prime} \sigma \mathrm{d} W_{s}
$$

with $\widehat{r}_{t}=\beta+\left(r-\alpha \beta P_{t}\right) /\left(1+\beta P_{t}\right), Q_{t-s}^{\prime}=\mathrm{d} Q_{t-s} / \mathrm{d} t$, and $P_{t}$ and $Q_{t-s}$ defined in (17) and 23 , respectively.

The right-hand side of Eqn. (26) consists of three terms. The first term $g_{t} \mathrm{~d} t$ represents the unconditional median growth rate of log consumption. Two counteracting forces determine how large the unconditional median growth rate is. First, the individual has

\footnotetext{
${ }^{31}$ This number corresponds to the increase in the S\&P 500 index between January 1, 2009 and December 31, 2009.
} 


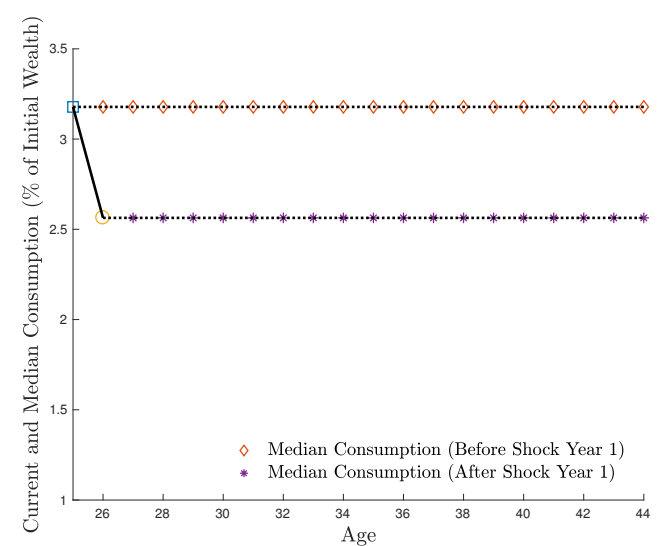

(a) CRRA Individual

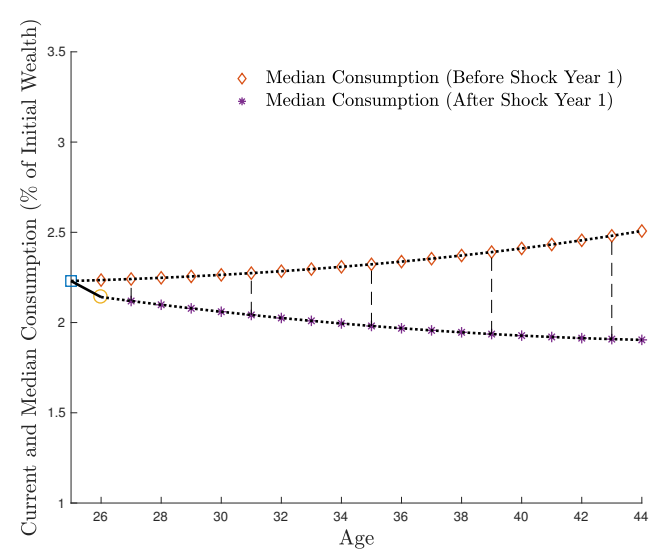

(b) Individual with Habit Preferences

$+24 \%$ stock return shock in year 2 (next to the $-38 \%$ stock return shock in year 1 )

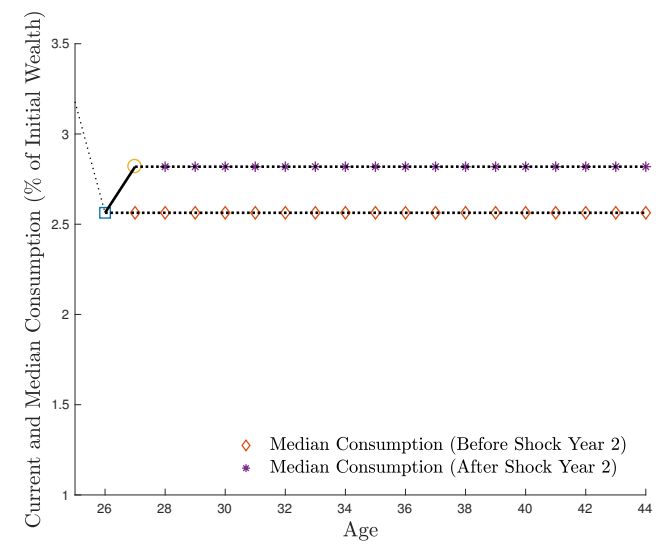

(c) CRRA Individual

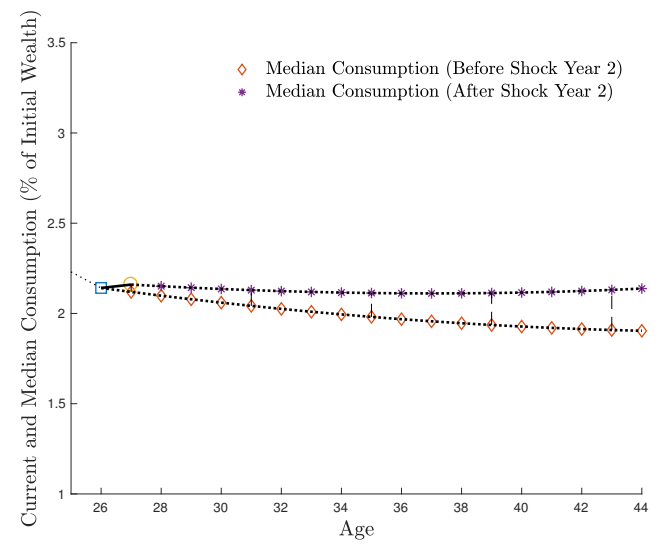

(d) Individual with Habit Preferences

Figure 2: Shock absorbing mechanisms. The figure shows the impact of stock return shocks on current and future consumption choices. The left panels consider a CRRA individual, while the right panels consider an individual whose preferences exhibit internal habit formation (with preference parameters $\gamma=10, \alpha=0.3$, and $\beta=0.3$ ). The small solid lines represent the change in current consumption as a result of the shock. The CRRA individual invests $50 \%$ of his accumulated wealth in the stock market (i.e., his relative risk aversion coefficient is equal to 2). Wealth at the age of 25 is for both individuals equal to 45 . We set the risk-free interest rate $r$ equal to $1 \%$, the market price of risk $\lambda$ to 0.2 , the stock return volatility $\sigma$ to $20 \%$, and the subjective rate of time preference $\delta$ to $3 \%$.

a preference to reduce current consumption (i.e., to increase the unconditional median growth rate of log consumption). Indeed, a decrease in current consumption dampens future habit levels. Furthermore, it increases expected investment earnings, because the individual will be able to save more. The values for the parameters $\alpha, \beta, r$ and $\lambda$ jointly determine the strength of the first force. Second, the individual has a preference to increase current consumption (i.e., to reduce the unconditional median growth rate of 
log consumption). Indeed, the individual is impatient: he prefers to consume sooner rather than later. The value for the preference parameter $\delta$ determines the strength of the second force. A large value for $\delta$ implies a relatively impatient individual. The second term $p_{t} \mathrm{~d} t$ represents past stock return shocks that the individual translates into the current median growth rate of log consumption. This term disappears if preferences do not exhibit internal habit formation (i.e., $\beta=0$, so that $Q_{\tau}=1$ for all $\tau$ ). Finally, the last term $\lambda /(\gamma \sigma) \cdot \sigma \mathrm{d} W_{t}$ corresponds to the current stock return shock that the individual directly translates into his current consumption level.

Figure 3 illustrates a consumption path for different types of individuals. As shown by this figure, the consumption stream of an individual with habit preferences is smoother than the consumption stream of a CRRA individual. As is well-known, an excessively smooth consumption stream is also consistent with aggregate consumption data (see, e.g., Flavin (1985), Deaton (1987), and Campbell and Deaton (1989)) and other behavioral models (see, e.g., Kőszegi and Rabin (2006, 2007, 2009), Pagel (2017), and Van Bilsen et al. (2017)).

\subsection{Optimal Portfolio Choice}

Theorem 4.2 presents the (approximate) optimal portfolio choice $\pi_{t}^{*}$.

Theorem 4.2. Consider an individual with lifetime utility (20) and habit formation process (8) who solves the consumption and portfolio choice problem (16). Then the (approximate) optimal portfolio choice $\pi_{t}^{*}$ is given by

$$
\pi_{t}^{*}=\int_{0}^{T-t} q_{\tau} \frac{V_{t, \tau}}{V_{t}} \mathrm{~d} \tau \cdot A_{t} .
$$

Here, $V_{t}=\int_{0}^{T-t} V_{t, \tau} \mathrm{d} \tau$ and $V_{t, \tau}$ denotes the market value at time $t$ of $c_{t+\tau}^{*}$. Appendix $A$ provides an explicit analytical expression for $V_{t, \tau}($ see (53)).

Figure 4 illustrates the portfolio strategy $\pi_{t}^{*} / A_{t}$ of an individual with habit preferences. The individual implements a life-cycle investment strategy: the share of accumulated wealth invested in the risky stock decreases as the individual ages. Indeed, the individual 


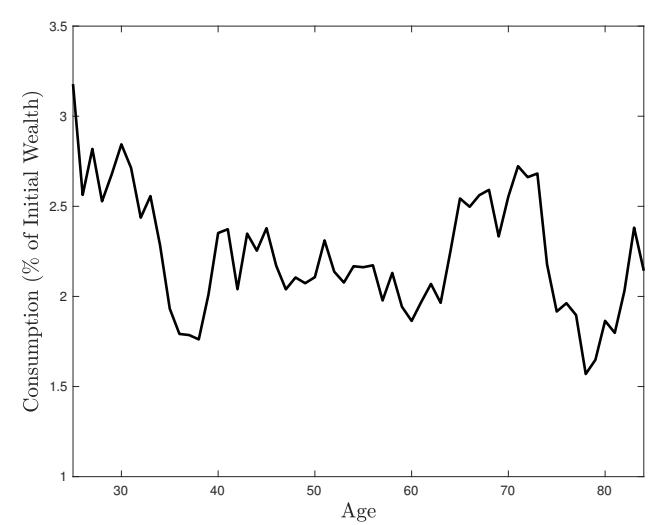

(a) CRRA Individual

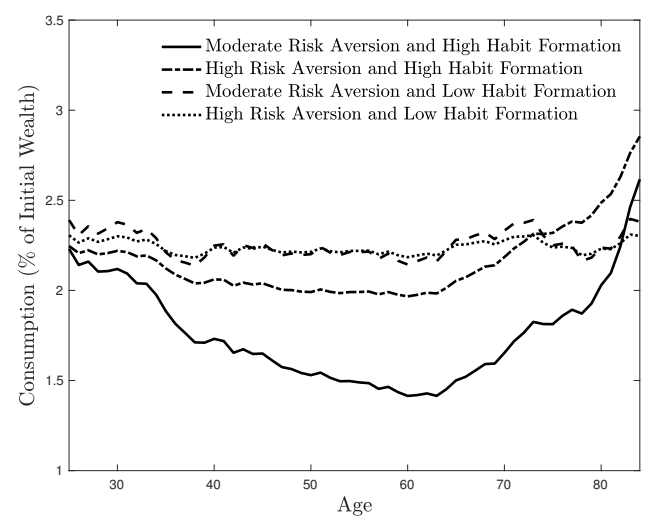

(b) Habit Preferences

Figure 3: Consumption dynamics. Panel A illustrates a consumption path of a CRRA individual, while panel B shows the impact of internal habit formation on the consumption dynamics. Panel B considers four different types of individuals: a moderately risk-averse individual with a high degree of habit persistence (i.e., $\gamma=10, \alpha=\beta=0.3$ ), a highly risk-averse individual with a high degree of habit persistence (i.e., $\gamma=20, \alpha=\beta=0.3$ ); a moderately risk-averse individual with a low degree of habit persistence (i.e., $\gamma=10, \alpha=0.2, \beta=0.1$ ), and a highly risk-averse individual with a low degree of habit persistence (i.e., $\gamma=20, \alpha=0.2, \beta=0.1$ ). The CRRA individual invests $50 \%$ of his accumulated wealth in the stock market (i.e., his relative risk aversion coefficient is equal to 2). Wealth at the age of 25 is for every individual equal to 45 . We set the risk-free interest rate $r$ equal to $1 \%$, the market price of risk $\lambda$ to 0.2 , the stock return volatility $\sigma$ to $20 \%$, and the subjective rate of time preference $\delta$ to $3 \%$. Individuals adjust consumption once a year.

has less time to absorb a stock return shock as he grows older. We observe that the larger the degree of habit persistence, the more pronounced the life-cycle investment strategy will be; see Figure $4(\mathrm{~b})$, A declining equity glide path during both the accumulation and the retirement phase is also commonly adopted by target date fund managers; see Morningstar $(2017)$. The portfolio strategy of an individual with habit preferences stands in sharp contrast to the portfolio strategy of a CRRA individual. Such an individual implements an age-independent portfolio strategy; see the dotted line in Figure $4(\mathrm{a}){ }^{32}$

Figure 4(a) also shows that the portfolio strategy of an individual with habit preferences hardly varies with the state of the economy, especially at higher ages ${ }^{33}$ The

\footnotetext{
${ }^{32}$ We note that a CRRA individual invests a constant share of total wealth, which equals the sum of financial wealth and human capital, in the risky stock.

${ }^{33}$ A state-independent portfolio strategy has three key advantages for annuity providers. First, an annuity provider can implement the portfolio strategy without much effort: he does not have to monitor any state variables. Second, an annuity with a state-independent portfolio strategy is easy to communicate as the equity glide path is known at inception. Third, the individual typically achieves a prosperous expected payout stream at an affordable price. Indeed, if an annuity provider offers an annuity with a state-dependent portfolio strategy, then this portfolio strategy is often designed such that it protects customers against losses or locks in investment gains. While attractive from the viewpoint of avoiding losses, the flip side of this investment behavior is that upward potential can be rather limited.
} 
portfolio strategy is not completely state-independent: while the sensitivity $q_{\tau}$ and volatility $\Sigma_{\tau}$ of future consumption are fully state-independent due to the constant relative risk aversion property, a shock to the economy alters the shape of the median consumption stream (see Figure 2). In particular, long horizons benefit relatively more from a positive shock, while, on the other hand, short horizons suffer relatively less from a negative shock. As a result, the value weights $V_{t, \tau} / V_{t}$ in (28) change following a shock. However, this effect is small (second-order), so that the portfolio strategy is nearly insensitive to economic shocks.

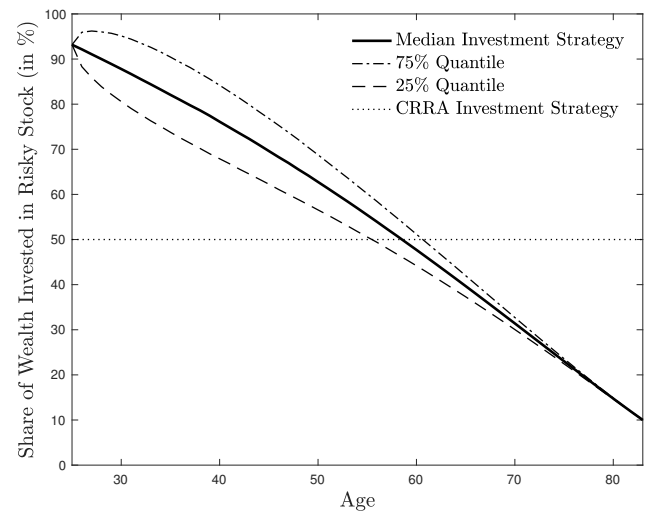

(a) Summary Statistics

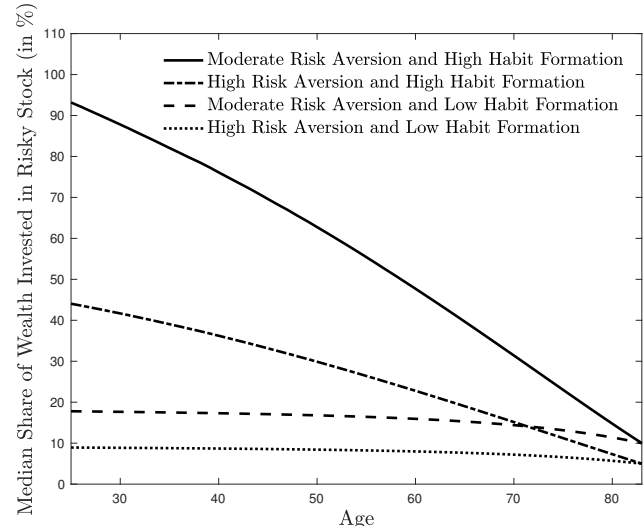

(b) Comparative Statics

Figure 4: Investment strategy. Panel A shows summary statistics of the investment strategy of an individual whose preferences exhibit internal habit formation (with preference parameters $\gamma=10$, $\alpha=0.3$, and $\beta=0.3$ ). Panel B illustrates how internal habit formation affects the median investment strategy. This panel considers four different types of individuals: a moderately risk-averse individual with a high degree of habit persistence (i.e., $\gamma=10, \alpha=\beta=0.3$ ), a highly risk-averse individual with a high degree of habit persistence (i.e., $\gamma=20, \alpha=\beta=0.3$ ); a moderately risk-averse individual with a low degree of habit persistence (i.e., $\gamma=10, \alpha=0.2, \beta=0.1$ ), and a highly risk-averse individual with a low degree of habit persistence (i.e., $\gamma=20, \alpha=0.2, \beta=0.1$ ). Wealth at the age of 25 is for every individual equal to 45 . We set the risk-free interest rate $r$ equal to $1 \%$, the market price of risk $\lambda$ to 0.2 , the stock return volatility $\sigma$ to $20 \%$, and the subjective rate of time preference $\delta$ to $3 \%$. Individuals adjust consumption once a year.

We note that the year-on-year consumption volatility is always equal to $2 \%$, irrespective of the individual's current age ${ }^{34}$ With ratio internal habit formation, the year-on-year consumption volatility is smaller than the year-on-year volatility of accumulated wealth. We find that the degree of habit persistence largely determines the share of accumulated wealth invested in the risky stock, while the individual's

\footnotetext{
${ }^{34}$ We note that the year-on-year consumption volatility is given by $\lambda /(\gamma \sigma) \cdot \sigma$ (see Eqn. 26$)$ ). Hence, assuming $\lambda=\sigma=0.2$ and $\gamma=10$, we find that the year-on-year consumption volatility is equal to $2 \%$.
} 
coefficient of relative risk aversion largely determines the degree of variability of current consumption. As a result, given a certain degree of relative risk aversion, an individual with habit preferences invests more in the stock market early in life than an individual with conventional CRRA preferences. An individual with habit preferences translates a stock return shock not only in current consumption but also in future growth rates of consumption. This enables the individual to take a relatively risky position in the stock market at young ages.

\subsection{Comparison with the Difference Internal Habit Model}

Several authors explore the optimal consumption and portfolio implications of the difference (or additive) internal habit model in which the individual derives utility from the difference between consumption and the habit level. More specifically, an individual with difference habit utility maximizes

$$
U(c-h)=\mathbb{E}\left[\int_{0}^{T} e^{-\delta t} \frac{1}{1-\gamma}\left(c_{t}-h_{t}\right)^{1-\gamma} \mathrm{d} t\right]
$$

subject to the budget constraint (7) and the dynamics $\mathrm{d} h_{t}=\left(\beta c_{t}-\alpha h_{t}\right) \mathrm{d} t$ with $h_{0} \geq 0$.

It follows from (29) that relative risk aversion is not constant - as in the ratio internal habit model - but rather depends on surplus consumption $c_{t}-h_{t}$. Also, under the difference habit model, the habit level is viewed as a minimum subsistence level ${ }^{35}$ while under the ratio habit model, the habit level is viewed as a standard-of-living. As a result, the optimal strategies under the difference habit model are considerably different from the optimal strategies under the ratio habit model; see Figure 5 which shows for both models the portfolio strategy at age 55 as a function of the log stock price index (Panel A) and the median portfolio strategy over time (Panel B). We have calibrated the difference habit model such that both models imply the same median portfolio strategy at age $55{ }^{36}$ As can be seen from Figure 5 (a), under the difference habit model, the individual

\footnotetext{
${ }^{35}$ The difference habit model requires an artificial constraint on the individual's initial wealth position or on the habit dynamics to avoid negative infinite utility.

${ }^{36}$ We solve the difference habit model using Schroder and Skiadas (2002). Furthermore, we choose the parameters describing the habit dynamics such that negative infinite utility is avoided.
} 
implements a relatively conservative investment strategy when the stock price index is low. Indeed, in bad states of the economy, wealth can be just sufficient to finance future habit levels. As they represent the minimum subsistence levels under the difference habit model, the individual becomes very risk averse. Furthermore, we observe from Figure 5(b) that under the ratio habit model, the share of wealth invested in the stock tends to decrease with age, while this life-cycle feature of the investment strategy is much less pronounced under the difference habit model. Indeed, under the difference habit model, wealth is first allocated to the risk-free asset to finance the minimum subsistence level. Any remaining wealth is invested according to the optimal portfolio strategy of the CRRA individual (see Merton (1969)).

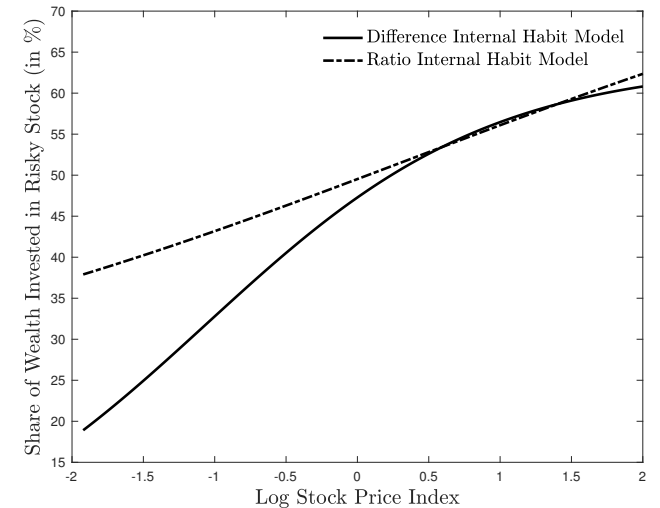

(a) Portfolio Strategy across States

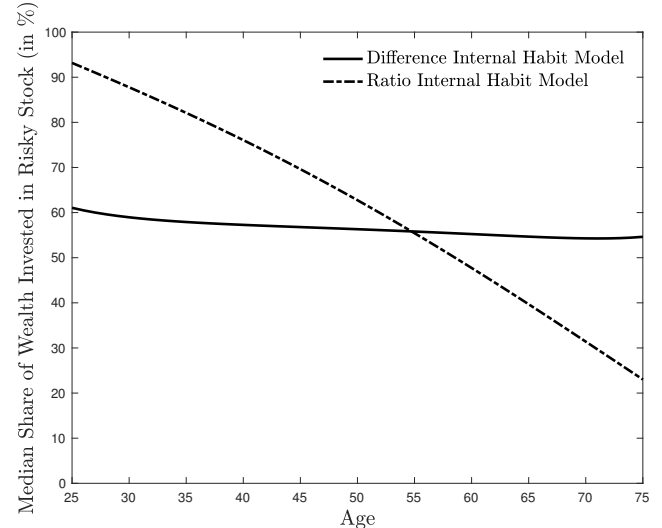

(b) Portfolio Strategy over Time

Figure 5: Portfolio strategy across states and over time. This figure illustrates the portfolio strategy at age 55 as a function of the log stock price index (Panel A) and the median portfolio strategy over time (Panel B) for both the difference habit model and the ratio habit model. In the case of the ratio habit model, the preference parameters are as follows: $\gamma=10$ and $\alpha=\beta=0.3$. We have calibrated the difference habit model such that both models imply the same median portfolio strategy at age 55 . We set the risk-free interest rate $r$ equal to $1 \%$, the market price of risk $\lambda$ to 0.2 , the stock return volatility $\sigma$ to $20 \%$, and the subjective time preference rate $\delta$ to $3 \%$. Individuals adjust consumption once a year.

Figure 6 shows that for both models the consumption-to-wealth ratio decreases as the stock price index goes down: current consumption responds less than one-to-one to a change in wealth. We also observe that the consumption-to-wealth ratio implied by the difference habit model can become relatively large in bad states of the economy when compared to the ratio habit model. Indeed, under the difference habit model, consumption can never fall below the habit level. 


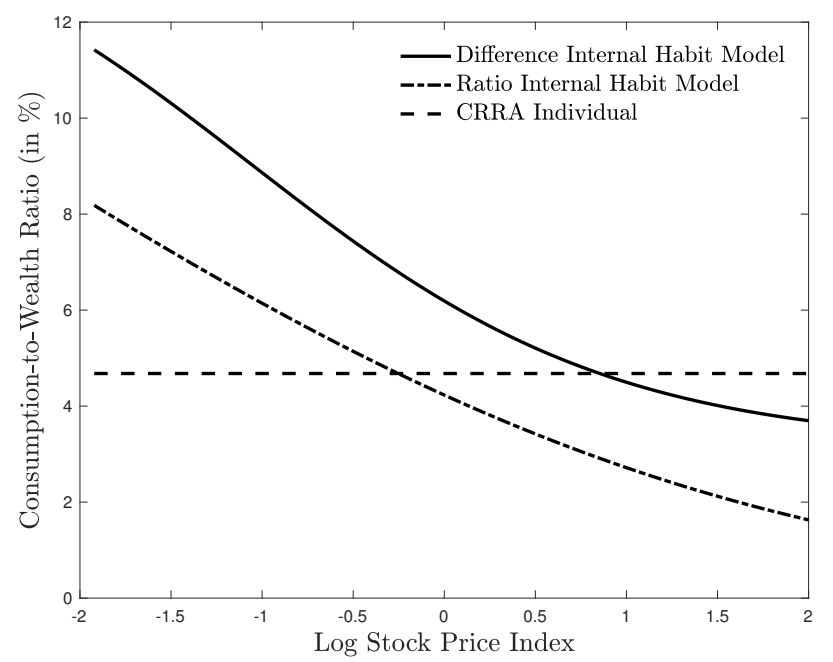

Figure 6: Consumption-to-wealth ratio. This figure shows the consumption-to-wealth ratio at age 55 as a function of the log stock price index for both the difference habit model and the ratio habit model. In the case of the ratio habit model, the preference parameters are as follows: $\gamma=10$ and $\alpha=\beta=0.3$. We have calibrated the difference habit model such that both models imply the same median portfolio strategy at age 55 . The CRRA individual invests $50 \%$ of his wealth in the stock market (i.e., his relative risk aversion coefficient equals 2). Wealth at age 25 is equal to 45 for each model. We set the risk-free interest rate $r$ equal to $1 \%$, the market price of risk $\lambda$ to 0.2 , the stock return volatility $\sigma$ to $20 \%$, and the subjective time preference rate $\delta$ to $3 \%$. Individuals adjust consumption once a year.

\section{$5 \quad$ Internal Habits and Stochastic Interest Rates}

This section explores the implications of a stochastic interest rate for the optimal consumption and portfolio choice of an individual with ratio internal habit preferences. We assume that the economy consists of three assets: one (locally) risk-free asset, a risky stock, and a risky zero-coupon bond with maturity date $T_{1}$. The price of the risk-free asset, $B_{t}$, and the $(2 \times 1)$-vector of risky asset prices, $S_{t}$, satisfy ${ }^{37}$

$$
\frac{\mathrm{d} B_{t}}{B_{t}}=r_{t} \mathrm{~d} t, \quad \frac{\mathrm{d} S_{t}}{S_{t}}=\mu_{t} \mathrm{~d} t+\sigma_{t} \mathrm{~d} W_{t}
$$

where the risk-free interest rate $r_{t}$ follows an Ornstein-Uhlenbeck process, i.e.,

$$
\mathrm{d} r_{t}=\kappa\left(\bar{r}-r_{t}\right) \mathrm{d} t+\left[\begin{array}{c}
\sigma_{r} \rho \\
\sigma_{r} \sqrt{1-\rho^{2}}
\end{array}\right]^{\top} \mathrm{d} W_{t},
$$

\footnotetext{
${ }^{37}$ Note that this economy arises as a special case of the one considered by Brennan and Xia (2002).
} 
and $\mu_{t}$ and $\sigma_{t}$ are defined as follows:

$$
\mu_{t}=\left[\begin{array}{c}
r_{t}+\lambda_{1} \sigma_{S} \\
r_{t}-\sigma_{r} D_{T_{1}-t}\left(\lambda_{1} \rho+\lambda_{2} \sqrt{1-\rho^{2}}\right)
\end{array}\right], \sigma_{t}=\left[\begin{array}{cc}
\sigma_{S} & 0 \\
-\sigma_{r} D_{T_{1}-t} \rho & -\sigma_{r} D_{T_{1}-t} \sqrt{1-\rho^{2}}
\end{array}\right] .
$$

Here, $\kappa \geq 0$ is the mean reversion coefficient, $\bar{r}$ is the long-term interest rate, $\sigma_{r}>0$ is the interest rate volatility, $-1 \leq \rho \leq 1$ models the correlation between the interest rate and the risky stock price, $\sigma_{S}>0$ represents the stock return volatility, and $D_{T_{1}-t}=$ $\frac{1}{\kappa}\left(1-e^{-\kappa\left(T_{1}-t\right)}\right)$ is the interest rate sensitivity of the bond. The market prices of risk associated with the Brownian increments are given by $\lambda_{1}$ and $\lambda_{2}$.

Appendix A proves the following theorem.

Theorem 5.1. Consider an individual with lifetime utility (20) and habit formation process (8) who solves the consumption and portfolio choice problem (16). Assume that the interest rate $r_{t}$ satisfies (31) and that the economy consists of a (locally) risk-free asset, a stock, and a zero-coupon bond with maturity date $T_{1}$. Let the dynamics of the assets be given by (30). Then the optimal amounts of wealth invested in the stock and bond are given by

$$
\begin{gathered}
\pi_{1, t}^{*}=-\frac{1}{\sigma_{S}} \frac{\partial V_{t}}{\partial \log \widehat{M}_{t}} \frac{1}{V_{t}} \cdot\left(\widehat{\lambda}_{1, t}-\frac{\rho}{\sqrt{1-\rho^{2}}} \widehat{\lambda}_{2, t}\right) \cdot A_{t} \\
\pi_{2, t}^{*}=\frac{1}{\sigma_{r} \sqrt{1-\rho^{2}} D_{T_{1}-t}} \frac{\partial V_{t}}{\partial \log \widehat{M}_{t}} \frac{1}{V_{t}} \cdot \widehat{\lambda}_{2, t} \cdot A_{t}-\frac{1}{D_{T_{1}-t}} \cdot \frac{\partial V_{t}}{\partial r_{t}},
\end{gathered}
$$

with $V_{t}=\int_{0}^{T-t} V_{t, \tau} \mathrm{d} \tau$ representing the market value of the future (approximate) optimal consumption stream $\left\{c_{s}^{*}\right\}_{t \leq s \leq T}$ and

$$
\widehat{\lambda}_{1, t}=\lambda_{1}+\beta \frac{\sigma_{r} \rho \widehat{D}_{t} P_{t}}{1+\beta P_{t}}, \quad \widehat{\lambda}_{2, t}=\lambda_{2}+\beta \frac{\sigma_{r} \sqrt{1-\rho^{2}} \widehat{D}_{t} P_{t}}{1+\beta P_{t}}
$$

with $\widehat{M}_{t} \equiv M_{t}\left(1+\beta P_{t}\right), P_{t}$ given by 17 and $\widehat{D}_{t}$ defined in Appendix $A$ (see 60 ).

Figure $7(\mathrm{a})$ shows the first component of the bond portfolio weight $\pi_{2, t}^{*} / A_{t}$ (see (34)) as a function of age. We call this component the speculative bond portfolio weight. Two counteracting forces determine how this speculative weight evolves over the individual's 
life cycle. On the one hand, the available time to incorporate a speculative shock into future consumption declines with age. As a result, the speculative demand decreases as the individual becomes older. A similar reasoning applies to the stock portfolio weight; see Section 4.5. On the other hand, the older the individual, the more sensitive the individual's relative consumption choice $\widehat{c}_{t}^{*}$ (typically) is to interest rate shocks; see Eqn. (63) in Appendix A which shows that $\widehat{\lambda}_{2, t} / \gamma$ models the interest rate sensitivity of $\widehat{c}_{t}^{*}$. Note that $\widehat{\lambda}_{2, t}$ becomes more negative as the individual ages. This causes the speculative demand to increase with age. The first effect dominates the second effect in Figure 7 (a).

Figure $7(\mathrm{~b})$ shows the second component of the bond portfolio weight $\pi_{2, t}^{*} / A_{t}$ (see again (34) ) as a function of age. We call this component the hedging bond portfolio weight. The value of the hedging weight is also the result of two counteracting forces: a horizon effect and a substitution effect. On the one hand, the longer the horizon $\tau$, the larger the impact of a shock in the interest rate will be on the price of future consumption. This causes the hedging portfolio weight to decrease over the life cycle. On the other hand, we find a new effect that causes the hedging bond portfolio weight to increase over the life cycle. We can explain this effect by the fact that the willingness of a habit-forming individual to substitute consumption over time decreases with age. Indeed, as the individual ages, the duration of remaining lifetime consumption declines, and hence the current habit level determines to a greater extent future consumption levels. Jointly, these two effects lead to a hump-shaped pattern. Finally, we note that the second effect may explain why not many young individuals include long-terms bonds in their investment portfolios; see Morningstar (2017).

\section{Internal Habits and Epstein-Zin Utility}

As shown in Appendix B, an individual with habit preferences prefers (unrealistically) high unconditional median growth rates of log consumption (especially at high ages) except when his time preference rate $\delta$ is excessive ${ }^{38}$ This section therefore considers a utility specification that disentangles the elasticity of intertemporal

\footnotetext{
${ }^{38}$ Indeed, as already pointed out by Deaton (1992), an individual with habit preferences derives utility not only from consumption levels but also from consumption growth.
} 


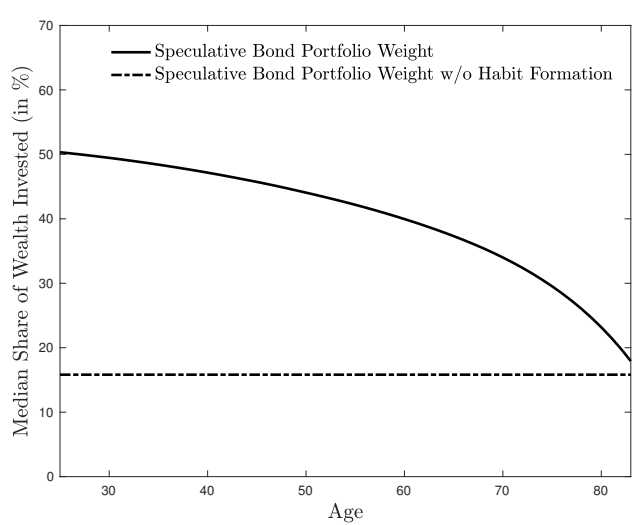

(a) Speculative Bond Portfolio Weights

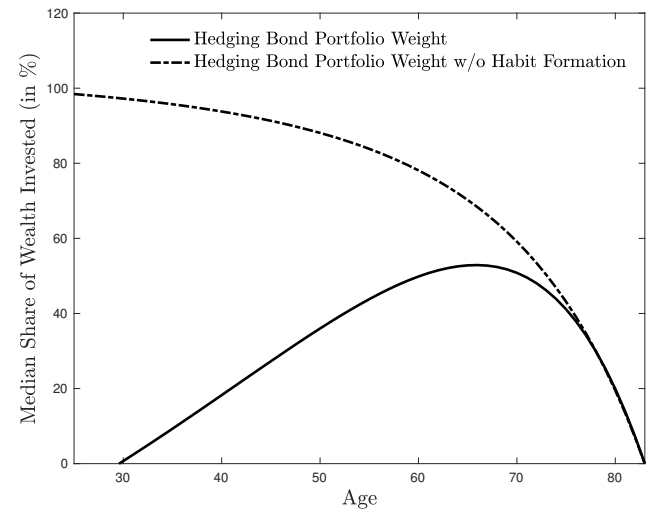

(b) Hedging Bond Portfolio Weights

Figure 7: Portfolio choice with stochastic interest rates. Panel A shows the median speculative bond portfolio weight (with and without habit formation) as a function of age. We assume that the individual invests wealth in a zero-coupon bond with a fixed time to maturity of 10 (i.e., $T_{1}-t=10$ ). Panel B shows the median hedging bond portfolio weight (with and without habit formation) as a function of age. The individual's preference parameters are as follows: $\gamma=10, \alpha=0.3$, and $\beta=0.3$ (for the case of no habit formation, we have $\alpha=\beta=0$ ). We set the long-term interest rate $\bar{r}$ equal to $1 \%$, the mean reversion parameter $\kappa$ to 0.1 , the interest rate volatility $\sigma_{r}$ to $2 \%$, the market price of interest rate risk $\lambda_{2}$ to -0.2 , the market price of stock market risk $\lambda_{1}$ to 0.2 , the stock return volatility $\sigma_{S}$ to $20 \%$, and the subjective rate of time preference $\delta$ to $3 \%$. The individual adjusts consumption once a year. Note that part of the individual's wealth is invested in the money market account.

substitution from the coefficient of relative risk aversion. Under this extended preference model, quite remarkably, median consumption growth can be low or moderate even when the individual's time preference rate $\delta$ takes on reasonable values.

\subsection{Utility Specification}

We consider an individual with Epstein-Zin utility in terms of relative consumption. Let $\left\{U_{t}\right\}_{0 \leq t \leq T}$ be the utility process. We assume that $\left\{U_{t}\right\}_{0 \leq t \leq T}$ satisfies the following integral equation $(0 \leq t \leq T)$ :

$$
U_{t}\left(\frac{c}{h}\right)=\mathbb{E}_{t}\left[\int_{t}^{T} f\left(\frac{c_{s}}{h_{s}}, U_{s}\right) \mathrm{d} s\right]
$$


Here, $\mathbb{E}_{t}$ denotes the expectation conditional upon information at time $t$. The intertemporal aggregator $f$ is assumed to be given by ${ }^{39}$

$$
f\left(\frac{c_{t}}{h_{t}}, U_{t}\right)=(1+\zeta)\left[\frac{\left(\frac{c_{t}}{h_{t}}\right)^{\varphi}}{\varphi}\left|U_{t}\right|^{\frac{\zeta}{1+\zeta}}-\delta U_{t}\right]
$$

Here, $\zeta>-1$ and $\varphi<\min \{1,1 /(1+\zeta)\}$ are preference parameters. We refer to (37) as the Kreps-Porteus aggregator (Kreps and Porteus (1978)) ${ }^{40}$ The individual maximizes $U_{0}\left(\frac{c}{h}\right)$ (see (36) ) with $f\left(\frac{c_{t}}{h_{t}}, U_{t}\right)$ given by (37) subject to the habit process (8) and the dynamic budget constraint (7).

\subsection{Dynamic Consumption and Portfolio Choice}

We can solve the individual's optimization problem by first invoking our pathwise approximation approach and next the approach of Schroder and Skiadas (1999). The following theorem presents the (approximate) optimal consumption choice.

Theorem 6.1. Consider an individual with utility process (36), intertemporal aggregator (37) and habit formation process (8) who solves the consumption and portfolio choice problem (16). Assume constant investment opportunities (i.e., $r_{t}=r, \mu_{t}=\mu, \sigma_{t}=\sigma$ and $\lambda_{t}=\lambda$ for all $\left.t\right)$. Let $h_{t}^{*}$ be the individual's habit level implied by substituting the individual's optimal past relative consumption choices $\widehat{c}_{s}^{*}(s \leq t)$ into (13) and let $z$ be a scaling parameter associated with the static budget constraint in (16). Then the individual's (approximate) optimal consumption choice $c_{t}^{*}$ is given by

$$
c_{t}^{*}=h_{t}^{*} z \exp \left\{\int_{0}^{t}\left(\psi\left[\widehat{r}_{s}+\frac{1}{2} \frac{\lambda^{2}}{\gamma}-\delta\right]+\frac{1}{2} \frac{\lambda^{2}(\gamma-1)}{\gamma^{2}}\right) \mathrm{d} s+\frac{\lambda}{\gamma} \int_{0}^{t} \mathrm{~d} W_{s}\right\},
$$

${ }^{39}$ If $\varphi=0$, then (37) reduces to $f\left(c_{t} / h_{t}, U_{t}\right)=\left(1+\zeta U_{t}\right)\left[\log \left\{c_{t} / h_{t}\right\}-(\delta / \zeta) \log \left\{1+\zeta U_{t}\right\}\right]$.

${ }^{40}$ If $\zeta=0$ and the habit level $h_{t}$ equals unity (i.e., $\left.\alpha=\beta=0\right)$, then $f\left(c_{t} / h_{t}, U_{t}\right)$ reduces to

$$
f\left(\frac{c_{t}}{h_{t}}, U_{t}\right)=\frac{1}{\varphi} c_{t}^{\varphi}-\delta U_{t}
$$

Eqn. (36) is then equivalent to the additive utility specification

$$
U_{t}\left(\frac{c}{h}\right)=\mathbb{E}_{t}\left[\int_{t}^{T} e^{-\delta(s-t)} \frac{1}{\varphi} c_{s}^{\varphi} \mathrm{d} s\right] .
$$


where $\psi=1 /(1-\varphi)$ and $\gamma=1-\varphi(1+\zeta)$. The scaling parameter $z \geq 0$ is determined such that the individual's original budget constraint holds with equality.

From 40 one may verify that the sensitivity $q_{\tau}$ and volatility $\Sigma_{\tau}$ of future consumption take the same form as in the base-line model (see Section 4). In the preference model of this section, the parameter $\psi$ models the individual's willingness to substitute consumption over time. Relative risk aversion is thus decoupled from the elasticity of intertemporal substitution. Figure 8 illustrates the median consumption path as a function of age for an individual whose preferences combine Epstein-Zin utility with the ratio internal habit model. As in Section 4, we assume $\alpha=\beta=0.3$ and $\gamma=10$. Figure 8 shows that the growth rates of the individual's median consumption path are substantially lower at high ages compared to the case without Epstein-Zin utility. Indeed, if the elasticity of intertemporal substitution is relatively low (as is the case in Figure 8 where $\psi$ equals zero), the individual is less willing to substitute current consumption for future consumption in order to avoid large future habit levels.

The general expression for the (approximate) optimal portfolio choice under EpsteinZin utility in an economy with one risky asset remains the same as in Section 4, see, in particular, Eqn. (28). However, under Epstein-Zin utility, long horizons receive smaller value weights in the computation of the portfolio strategy compared to the case without Epstein-Zin utility, as wealth accumulation during retirement is not excessive. As a result, an individual whose preferences combine Epstein-Zin utility with habit formation invests less in the risky stock than an individual whose preferences are described by the ratio internal habit model without Epstein-Zin utility; see Figure 9 which shows the reduction in the share of wealth invested in the risky stock as a result of superimposing Epstein-Zin utility to our base-line model.

\section{Accuracy of the Approximation Method}

The consumption and portfolio strategies presented in Sections 4,5 and 6 are exact only in the case when $\beta=0$ and/or $\alpha=\infty$. In all other cases, the consumption and 


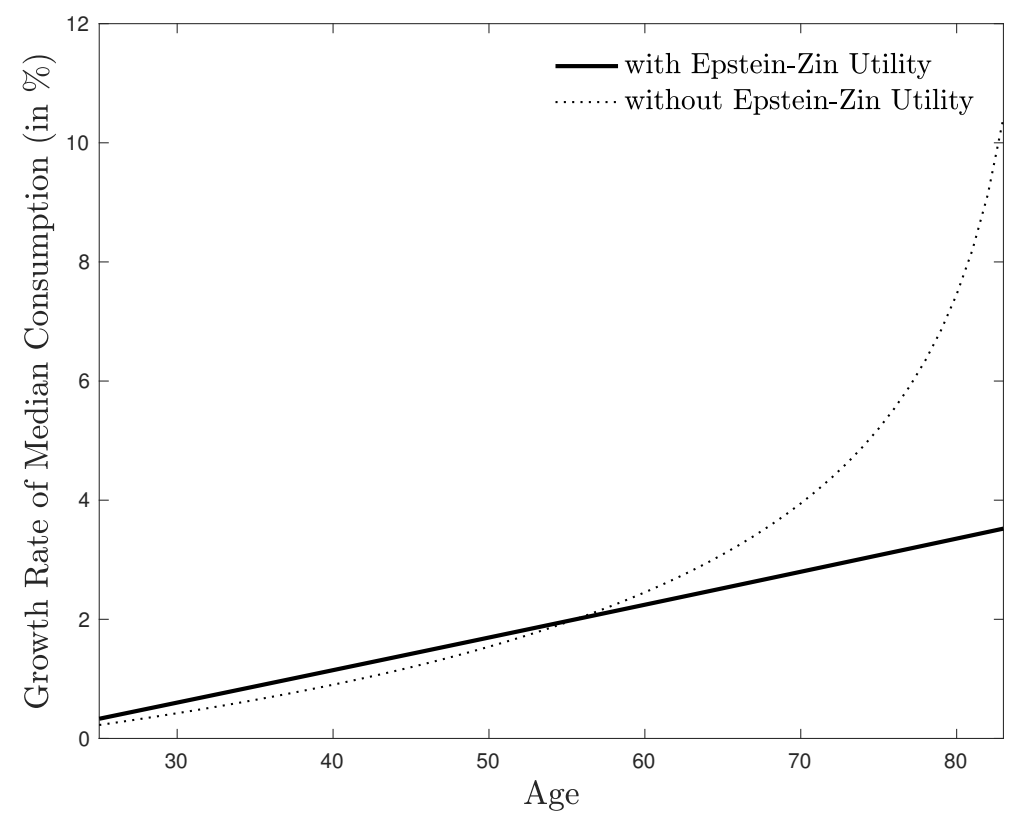

Figure 8: Median consumption path. The figure illustrates the median consumption path as a function of age for an individual whose preferences combine Epstein-Zin utility with the ratio internal habit model. The preference parameters are: $\psi=0, \gamma=10, \alpha=0.3$ and $\beta=0.3$. Wealth at the age of 25 is equal to 45 . For comparison purposes, we also plot the median consumption path for the case without Epstein-Zin utility; see the dotted line. We set the risk-free interest rate $r$ equal to $1 \%$, the market price of risk $\lambda$ to 0.2 , the stock return volatility $\sigma$ to $20 \%$, and the subjective rate of time preference $\delta$ to $3 \%$. The individual adjusts consumption once a year.

portfolio strategies are approximate based upon linearizing the individual's static budget constraint in (14) around the relative consumption trajectory $\left\{\widehat{c}_{t}\right\}_{0 \leq t \leq T}=1$. This section analyzes the approximation error induced by applying a pathwise linearization to the static budget constraint.

We consider an individual whose preferences are represented by (36) with aggregator (37) and habit formation process (8). We determine the genuine optimal consumption choice $c_{t}^{\text {opt }}$ and optimal portfolio choice $\pi_{t}^{\text {opt }}$ by using the method of backward induction; Appendix C provides details on the numerical solution technique. We evaluate the performance of the approximate optimal consumption choice $c_{t}^{*}$ by measuring the relative decline in certainty equivalent consumption ${ }^{41}$ Table 11 reports

\footnotetext{
${ }^{41}$ The certainty equivalent of an uncertain consumption strategy is defined to be the constant consumption level that yields indifference to the uncertain consumption strategy. The certainty equivalent consumption choice $c e$ always exists if $\alpha \geq \beta$. In particular, lifetime utility $U(c / h)$ is increasing in certainty equivalent consumption $c e$ if $\beta \int_{0}^{T} e^{-\alpha t} \mathrm{~d} t \leq 1$. If $T$ is large, then $\int_{0}^{T} e^{-\alpha t} \mathrm{~d} t \approx \frac{1}{\alpha}$. Hence, we can always compute (for any $T$ ) the certainty equivalent consumption choice $c e$ if $\frac{\beta}{\alpha} \leq 1$.
} 


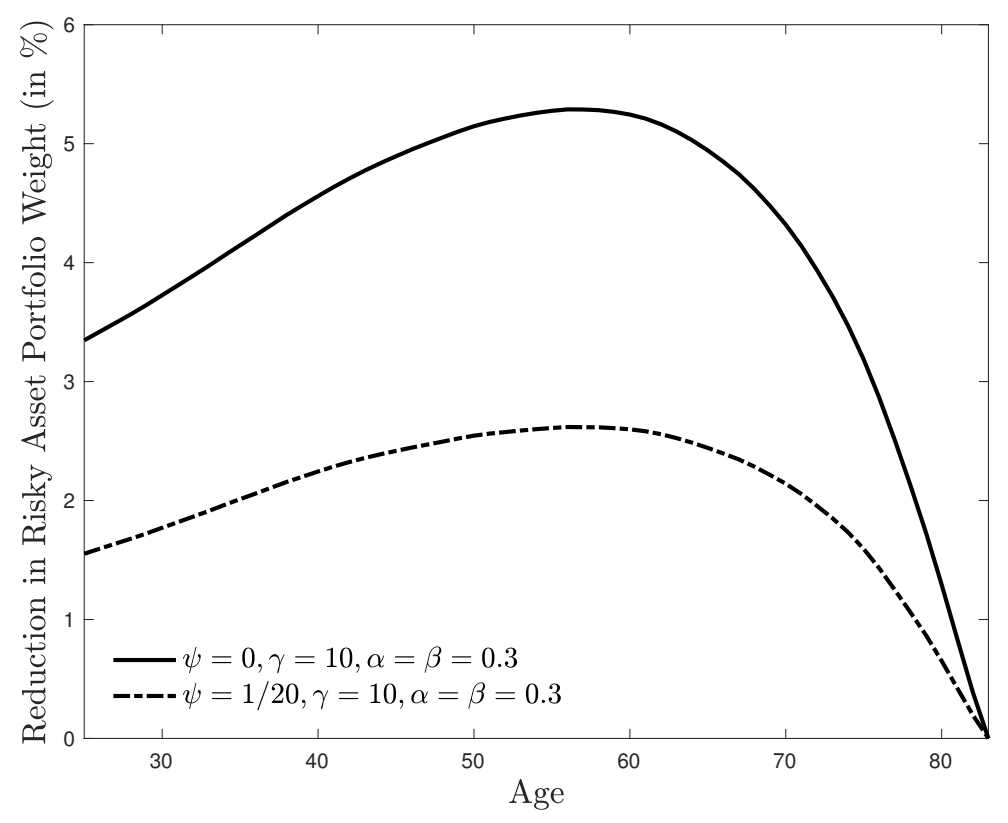

Figure 9: Reduction in risky stock portfolio weight. The figure shows the reduction in the share of wealth invested in the risky stock (in \%) as a result of superimposing Epstein-Zin utility to our baseline model as a function of age. The preference parameters are: $\psi=0, \gamma=10, \alpha=\beta=0.3$ (solid line) and $\psi=1 / 20, \gamma=10, \alpha=\beta=0.3$ (dash-dotted line). Wealth at the age of 25 is equal to 45 . We set the risk-free interest rate $r$ equal to $1 \%$, the market price of risk $\lambda$ to 0.2 , the stock return volatility $\sigma$ to $20 \%$, and the subjective time preference rate $\delta$ to $3 \%$. The individual adjusts consumption once a year.

our results. We find that the approximation error is a decreasing function of $\gamma$, and an increasing function of $\beta$. Indeed, if $\gamma$ is large, the habit level closely tracks consumption. Also, if $\beta$ is small, habit formation is rather limited. In nearly all cases, the approximation error is smaller than 1\%. Furthermore, we note that Table 1 only considers cases for which $\alpha$ equals $\beta$. If $\beta$ is smaller than $\alpha$, the welfare loss will be lower. In particular, in the limiting case $\beta=0$, the welfare loss will vanish. For illustration purposes, Figure 10 also compares, for three different economic scenarios, the optimal consumption path with the approximate consumption path. We observe a close match. To assess the accuracy of the approximation method further, Figure 11 shows $c_{t}^{\text {opt }} / h_{t}^{\text {opt }}$ for various sets of parameter values. We note that if $c_{t}^{\text {opt }} / h_{t}^{\text {opt }}$ is close to one, the approximation error is small. We find that the histograms are centered around one and that, as expected, the histogram width decreases when $\gamma$ goes up.

Finally, we compute the minimum welfare loss associated with implementing the Merton consumption strategy (Merton (1969)). This consumption strategy is 


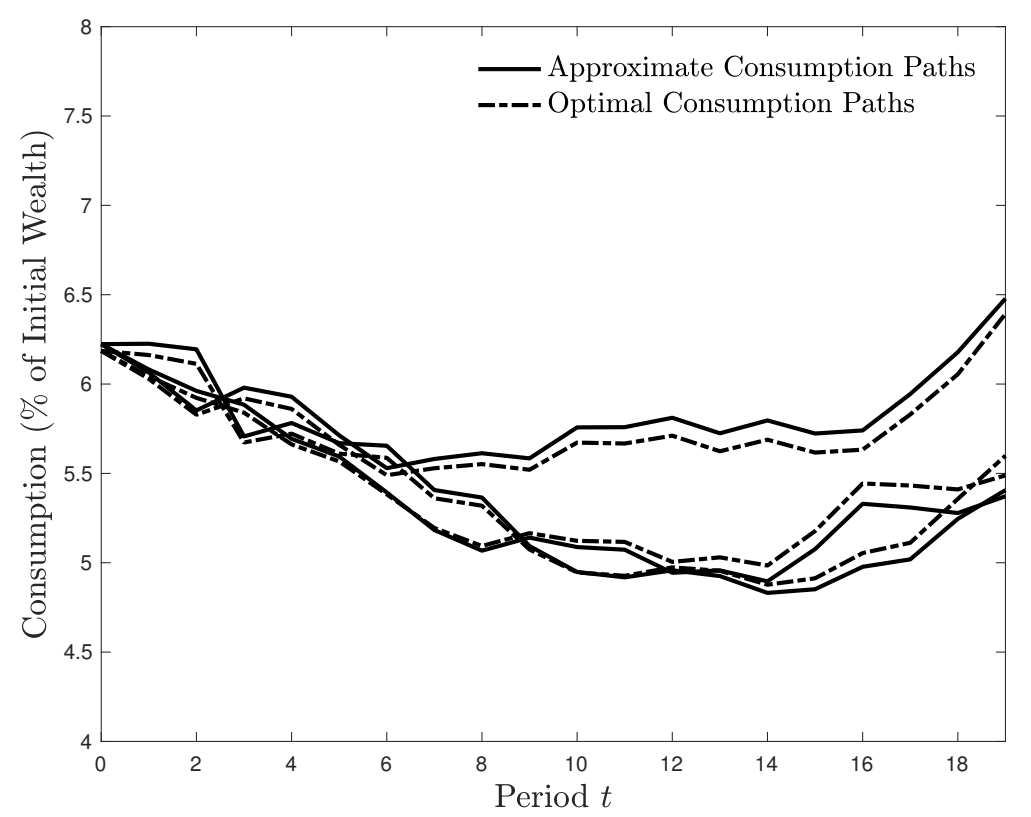

Figure 10: Consumption trajectories. The figure compares, for three different scenarios, the optimal consumption path with the approximate consumption path. The preference parameters are: $\psi=1 / 10$, $\gamma=10, \alpha=0.3$ and $\beta=0.3$. Initial wealth equals 15 . We set the terminal time $T$ equal to 20 , the risk-free interest rate $r$ equal to $1 \%$, the market price of risk $\lambda$ to 0.2 , the stock return volatility $\sigma$ to $20 \%$, and the subjective time preference rate $\delta$ to $3 \%$. The individual adjusts consumption once a year.

characterized by the degree of relative risk aversion of the Merton individual. We assume that the habit-forming individual is restricted to implement the Merton consumption strategy. He chooses the relative risk aversion coefficient in the Merton model such that the difference between his utility associated with implementing the Merton consumption strategy and his utility associated with implementing the approximate optimal consumption strategy is minimal. Table 2 reports our results for various sets of parameter values. We find that the minimum welfare loss due to the Merton consumption strategy is likely a factor 10 larger than the welfare loss associated with our approximation method.

\section{Concluding Remarks}

This paper has explored how an individual who derives utility from the ratio between his consumption and an endogenous habit should consume and invest over the life cycle. It is well-known that analytical closed-form solutions to multiplicative internal habit 


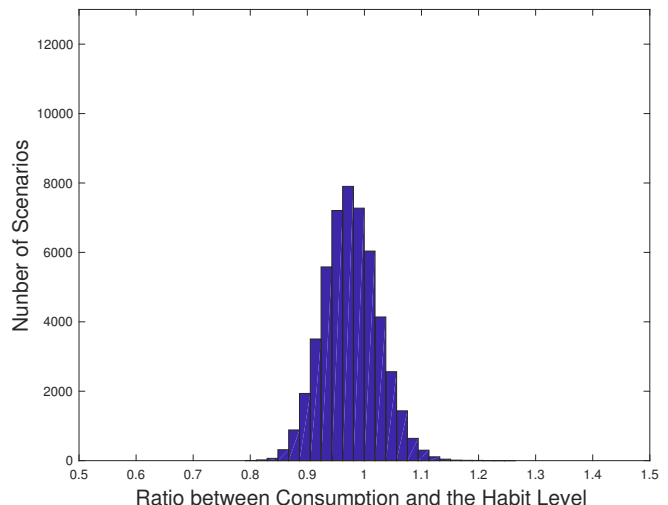

(a) $\gamma=10, \alpha=\beta=0.3$

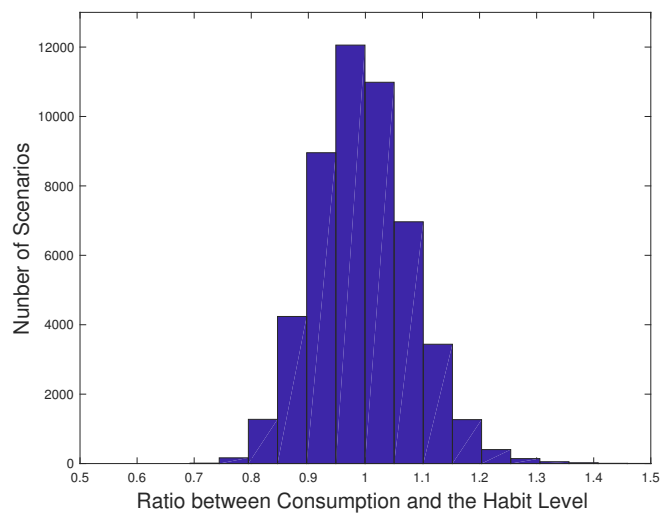

(c) $\gamma=5, \alpha=\beta=0.3$

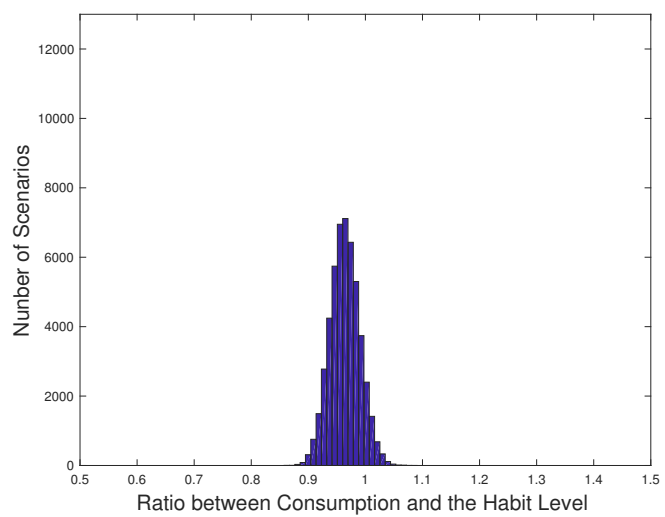

(e) $\gamma=20, \alpha=\beta=0.3$

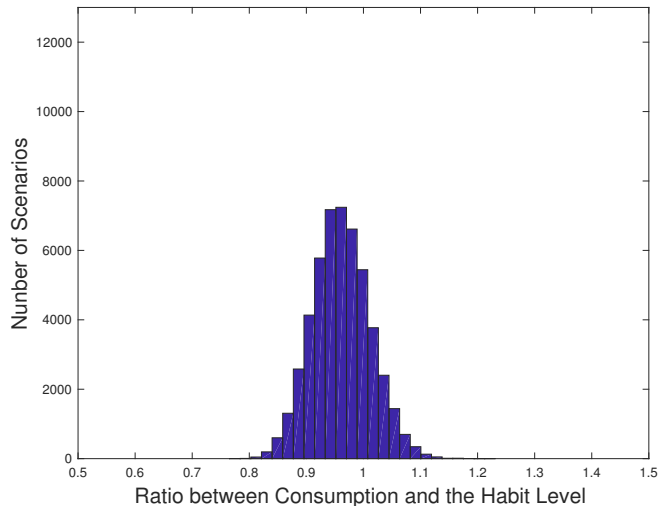

(b) $\gamma=10, \alpha=\beta=0.2$

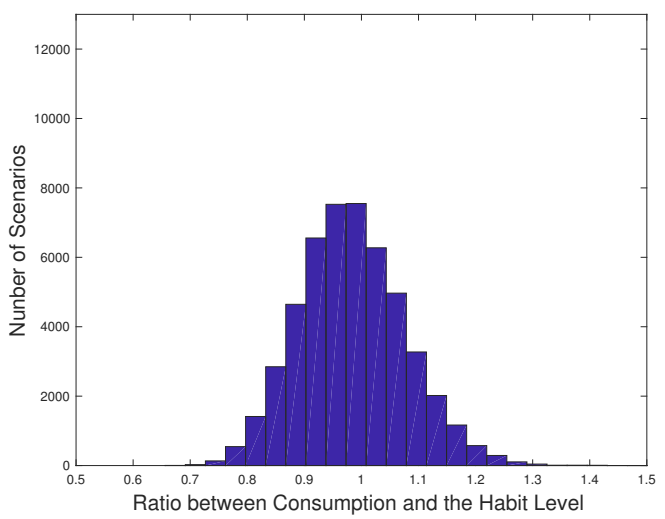

(d) $\gamma=5, \alpha=\beta=0.2$

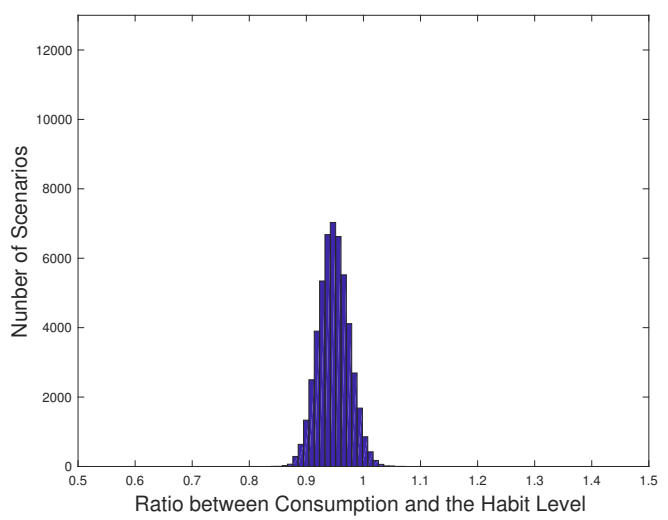

(f) $\gamma=20, \alpha=\beta=0.2$

Figure 11: Accuracy of the pathwise approximation method. The figure illustrates $c_{t}^{\mathrm{opt}} / h_{t}^{\mathrm{opt}}$ for various sets of parameter values. We assume $t=10$. We set the terminal time $T$ equal to 20 , the individual's initial wealth to 15 , the risk-free interest rate $r$ to $1 \%$, the market price of risk $\lambda$ to 0.2 , the stock return volatility $\sigma$ to $20 \%$, and the subjective rate of time preference $\delta$ to $3 \%$.

models do not exist in general. Therefore, we have developed a general solution procedure based on a linearization of the static budget constraint around the endogenous habit level 


\begin{tabular}{ccccccc}
\hline$\gamma$ & $\psi$ & $\alpha$ & $\beta$ & $\delta$ & $A_{0}$ & Welfare Loss (\%) \\
\hline 6 & $1 / 6$ & 0.30 & 0.30 & 0.03 & 15 & 1.0012 \\
8 & $1 / 8$ & 0.30 & 0.30 & 0.03 & 15 & 0.3967 \\
$\mathbf{1 0}$ & $1 / 10$ & $\mathbf{0 . 3 0}$ & $\mathbf{0 . 3 0}$ & $\mathbf{0 . 0 3}$ & $\mathbf{1 5}$ & $\mathbf{0 . 1 9 2 6}$ \\
12 & $1 / 12$ & 0.30 & 0.30 & 0.03 & 15 & 0.1006 \\
14 & $1 / 14$ & 0.30 & 0.30 & 0.03 & 15 & 0.0642 \\
\hline
\end{tabular}

(a) Sensitivity with respect to the Relative Risk Aversion Coefficient $\gamma$

\begin{tabular}{ccccccc}
\hline$\gamma$ & $\psi$ & $\alpha$ & $\beta$ & $\delta$ & $A_{0}$ & Welfare Loss (\%) \\
\hline 10 & $1 / 10$ & 0.20 & 0.20 & 0.03 & 15 & 0.0764 \\
10 & $1 / 10$ & 0.25 & 0.25 & 0.03 & 15 & 0.1237 \\
$\mathbf{1 0}$ & $1 / 10$ & $\mathbf{0 . 3 0}$ & $\mathbf{0 . 3 0}$ & $\mathbf{0 . 0 3}$ & $\mathbf{1 5}$ & $\mathbf{0 . 1 9 2 6}$ \\
10 & $1 / 10$ & 0.35 & 0.35 & 0.03 & 15 & 0.2827 \\
10 & $1 / 10$ & 0.40 & 0.40 & 0.03 & 15 & 0.3826 \\
\hline
\end{tabular}

(b) Sensitivity with respect to the Degree of Habit Formation $\alpha=\beta$

\begin{tabular}{ccccccc}
\hline$\gamma$ & $\psi$ & $\alpha$ & $\beta$ & $\delta$ & $A_{0}$ & Welfare Loss (\%) \\
\hline 10 & $1 / 10$ & 0.30 & 0.30 & 0.01 & 15 & 0.2090 \\
10 & $1 / 10$ & 0.30 & 0.30 & 0.02 & 15 & 0.2037 \\
$\mathbf{1 0}$ & $\mathbf{1 / 1 0}$ & $\mathbf{0 . 3 0}$ & $\mathbf{0 . 3 0}$ & $\mathbf{0 . 0 3}$ & $\mathbf{1 5}$ & $\mathbf{0 . 1 9 2 6}$ \\
10 & $1 / 10$ & 0.30 & 0.30 & 0.04 & 15 & 0.1897 \\
10 & $1 / 10$ & 0.30 & 0.30 & 0.05 & 15 & 0.1783 \\
\hline
\end{tabular}

(c) Sensitivity with respect to the Time Discount Rate $\delta$

\begin{tabular}{ccccccc}
\hline$\gamma$ & $\psi$ & $\alpha$ & $\beta$ & $\delta$ & $A_{0}$ & Welfare Loss (\%) \\
\hline 10 & $1 / 10$ & 0.30 & 0.30 & 0.03 & 13 & 0.1927 \\
10 & $1 / 10$ & 0.30 & 0.30 & 0.03 & 14 & 0.1881 \\
$\mathbf{1 0}$ & $1 / 10$ & $\mathbf{0 . 3 0}$ & $\mathbf{0 . 3 0}$ & $\mathbf{0 . 0 3}$ & $\mathbf{1 5}$ & $\mathbf{0 . 1 9 2 6}$ \\
10 & $1 / 10$ & 0.30 & 0.30 & 0.03 & 16 & 0.2081 \\
10 & $1 / 10$ & 0.30 & 0.30 & 0.03 & 17 & 0.2610 \\
\hline
\end{tabular}

(d) Sensitivity with respect to the Initial Wealth Level $A_{0}$

\begin{tabular}{ccccccc}
\hline$\gamma$ & $\psi$ & $\alpha$ & $\beta$ & $\delta$ & $A_{0}$ & Welfare Loss $(\%)$ \\
\hline 10 & $1 / 14$ & 0.30 & 0.30 & 0.03 & 15 & 0.2773 \\
10 & $1 / 12$ & 0.30 & 0.30 & 0.03 & 15 & 0.2506 \\
$\mathbf{1 0}$ & $1 / 10$ & $\mathbf{0 . 3 0}$ & $\mathbf{0 . 3 0}$ & $\mathbf{0 . 0 3}$ & $\mathbf{1 5}$ & $\mathbf{0 . 1 9 2 6}$ \\
10 & $1 / 8$ & 0.30 & 0.30 & 0.03 & 15 & 0.4286 \\
10 & $1 / 6$ & 0.30 & 0.30 & 0.03 & 15 & 1.2682 \\
\hline
\end{tabular}

(e) Sensitivity with respect to the Preference Parameter $\psi$

Table 1: Welfare losses. The table reports the welfare losses (in terms of the relative decline in certainty equivalent consumption) associated with implementing the approximate optimal consumption choice (21). We set the terminal time $T$ equal to 20 , the risk-free interest rate $r$ to $1 \%$, the market price of risk $\lambda$ to 0.2 , and the stock return volatility $\sigma$ to $20 \%$. The individual adjusts consumption once a year.

enabling us to transform consumption and portfolio problems with multiplicative internal habits into approximate consumption and portfolio problems without habits.

We have applied our general solution procedure to three important cases of 


\begin{tabular}{ccccccc}
\hline$\gamma$ & $\psi$ & $\alpha$ & $\beta$ & $\delta$ & $A_{0}$ & Welfare Loss (\%) \\
\hline 6 & $1 / 6$ & 0.30 & 0.30 & 0.03 & 15 & 2.8751 \\
14 & $1 / 14$ & 0.30 & 0.30 & 0.03 & 15 & 2.5241 \\
$\mathbf{1 0}$ & $1 / 10$ & $\mathbf{0 . 3 0}$ & $\mathbf{0 . 3 0}$ & $\mathbf{0 . 0 3}$ & $\mathbf{1 5}$ & $\mathbf{2 . 3 0 6 1}$ \\
10 & $1 / 10$ & 0.20 & 0.20 & 0.03 & 15 & 1.3031 \\
10 & $1 / 10$ & 0.40 & 0.40 & 0.03 & 15 & 3.3414 \\
\hline
\end{tabular}

Table 2: Minimum welfare losses. The table reports the minimum welfare losses (in terms of the relative decline in certainty equivalent consumption) associated with implementing the Merton consumption strategy. We set the terminal time $T$ equal to 20 , the risk-free interest rate $r$ to $1 \%$, the market price of risk $\lambda$ to 0.2 , and the stock return volatility $\sigma$ to $20 \%$. The individual adjusts consumption once a year.

multiplicative habit formation. The first case considers constant investment opportunities and assumes that the agent has additive preferences in terms of relative consumption; see Section 4. We have shown that the agent's preferences induce clearly interpretable implications: the relative risk aversion coefficient controls the consumption volatility and the strength of habit persistence controls the extent to which a stock return shock impacts future growth rates of consumption. The second case is an extension that allows for stochastic interest rates and stock-bond investments; see Section 5. We have shown that the speculative bond portfolio weight declines with age and that the hedging bond portfolio weight displays a hump-shaped pattern over the life cycle. Finally, we have studied an agent whose preferences combine ratio internal habits with Epstein-Zin utility; see Section 6. Interestingly, median consumption no longer grows at high rates at high ages and risky assets become less attractive.

\section{A Proofs}

\section{A.1 Proof of Theorem 3.1}

This appendix discusses how to approximate the left-hand side of the new static budget constraint in (14) around the constant relative consumption trajectory $\widehat{c} \equiv\left\{\widehat{c}_{t}\right\}_{0 \leq t \leq T} \equiv 1$. We define the following function:

$$
F(\widehat{c}) \equiv \int_{0}^{T} M_{t} h_{t} \widehat{c}_{t} \mathrm{~d} t
$$


with $\widehat{c} \equiv\left\{\widehat{c}_{t}\right\}_{0 \leq t \leq T}$ the individual's relative consumption process, $M_{t}$ given by (5), and $h_{t}$ given by (13). By invoking Taylor's theorem up to the first order, we obtain

$$
F(\widehat{c}) \approx F(z)+\mathrm{d} F(z, \widehat{c}-z)
$$

where $\mathrm{d} F(z, \widehat{c}-z)$ denotes the Gateaux differential of $F$ at $z \equiv\left\{z_{t}\right\}_{0 \leq t \leq T} \equiv 1$ in the direction $\widehat{c}-z$, i.e.,

$$
\begin{aligned}
\mathrm{d} F(z, \widehat{c}-z) & =\lim _{\varepsilon \rightarrow 0} \frac{F(z+\varepsilon(\widehat{c}-z))-F(z)}{\varepsilon}=\left.\frac{\mathrm{d}}{\mathrm{d} \varepsilon} F(z+\varepsilon(\widehat{c}-z))\right|_{\varepsilon=0} \\
& =\left.\frac{\mathrm{d}}{\mathrm{d} \varepsilon} \int_{0}^{T} M_{t} e^{\beta \int_{0}^{t} e^{-(\alpha-\beta)(t-s)} \log \left[z_{s}+\varepsilon\left(\widehat{c}_{s}-z_{s}\right)\right] \mathrm{d} s}\left(z_{t}+\varepsilon\left(\widehat{c}_{t}-z_{t}\right)\right) \mathrm{d} t\right|_{\varepsilon=0}
\end{aligned}
$$

See, e.g., Duffie and Skiadas (1992) for applications of Gateaux differentiation to the calculation of gradients.

We find that

$$
\begin{gathered}
F(z)=\int_{0}^{T} M_{t} \mathrm{~d} t \\
\mathrm{~d} F(z, \widehat{c}-z)=\int_{0}^{T}\left[M_{t}+\beta\left(\int_{t}^{T} M_{s} e^{-(\alpha-\beta)(s-t)} \mathrm{d} s\right)\right]\left(\widehat{c}_{t}-1\right) \mathrm{d} t .
\end{gathered}
$$

Hence, we can approximate the left-hand side of the static budget constraint in (14) by

$$
\begin{aligned}
\mathbb{E}[F(\widehat{c})] & \approx \mathbb{E}[F(z)+\mathrm{d} F(z, \widehat{c}-z)] \\
& =\mathbb{E}\left[\int_{0}^{T} M_{t} \mathrm{~d} t+\int_{0}^{T}\left[M_{t}+\beta\left(\int_{t}^{T} M_{s} e^{-(\alpha-\beta)(s-t)} \mathrm{d} s\right)\right]\left(\widehat{c}_{t}-1\right) \mathrm{d} t\right] \\
& =\mathbb{E}\left[\int_{0}^{T} M_{t} \mathrm{~d} t+\int_{0}^{T} M_{t} \mathbb{E}_{t}\left\{\left[1+\beta\left(\int_{t}^{T} \frac{M_{s}}{M_{t}} e^{-(\alpha-\beta)(s-t)} \mathrm{d} s\right)\right]\left(\widehat{c}_{t}-1\right)\right\} \mathrm{d} t\right] \\
& =\mathbb{E}\left[\int_{0}^{T} M_{t} \mathrm{~d} t+\int_{0}^{T} M_{t}\left(1+\beta P_{t}\right)\left(\widehat{c}_{t}-1\right) \mathrm{d} t\right] \\
& =-\beta \mathbb{E}\left[\int_{0}^{T} M_{t} P_{t} \mathrm{~d} t\right]+\mathbb{E}\left[\int_{0}^{T} M_{t}\left(1+\beta P_{t}\right) \widehat{c}_{t} \mathrm{~d} t\right] .
\end{aligned}
$$

Here, $P_{t} \equiv \mathbb{E}_{t}\left[\int_{t}^{T} \frac{M_{s}}{M_{t}} e^{-(\alpha-\beta)(s-t)} \mathrm{d} s\right]$.

We can now establish the approximate optimization problem 16 as follows. 
1. First, we replace the left-hand side of the static budget constraint in 14 by 46 .

2. Second, we eliminate the constant term $-\beta \mathbb{E}\left[\int_{0}^{T} M_{t} P_{t} \mathrm{~d} t\right]$ from (46). This term does not play a role in determining the first-order optimality condition.

3. Finally, we redefine initial wealth $A_{0}$ to be $\widehat{A}_{0}$ such that the approximate optimal consumption strategy $\left\{c_{t}^{*}\right\}_{0 \leq t \leq T}=\left\{h_{t}^{*} \widehat{c}_{t}^{*}\right\}_{0 \leq t \leq T}$ is budget-feasible. That is,

$$
\mathbb{E}\left[\int_{0}^{T} M_{t} h_{t}^{*} \widehat{c}_{t}^{*} \mathrm{~d} t\right]=A_{0}
$$

Straightforward computations show that the initial wealth $\widehat{A}_{0}$ associated with the approximate problem is then given by

$$
\widehat{A}_{0}=A_{0}+\mathbb{E}\left[\int_{0}^{T} \widehat{M}_{t} \widehat{c}_{t}^{*} \mathrm{~d} t\right]-\mathbb{E}\left[\int_{0}^{T} M_{t} h_{t}^{*} \widehat{c}_{t}^{*} \mathrm{~d} t\right]
$$

Here, $\widehat{M}_{t} \equiv M_{t}\left(1+\beta P_{t}\right)$. Note that the value of $\widehat{A}_{0}$ can only be determined after the problem has been solved.

\section{A.2 Derivation of $(22)$ and $(26)$}

This appendix writes the individual's consumption choice $c_{t}^{*}$ in terms of unexpected past stock return shocks. We can write the stochastic discount factor $\widehat{M}_{t} \equiv M_{t}\left(1+\beta P_{t}\right)$ as follows (this follows from applying Itô's lemma to $\widehat{M}_{t}=f\left(M_{t}, P_{t}\right)$ ):

$$
\widehat{M}_{t}=\widehat{M}_{0} \exp \left\{-\int_{0}^{t}\left(\widehat{r}_{s}+\frac{1}{2} \lambda^{2}\right) \mathrm{d} s\right\} \exp \left\{-\lambda \int_{0}^{t} \mathrm{~d} W_{s}\right\}
$$

where $\widehat{r}_{s}=\beta+\frac{r-\alpha \beta P_{s}}{1+\beta P_{s}}$.

Substituting 49 into (21), we arrive at

$$
\widehat{c}_{t}^{*}=\frac{c_{t}^{*}}{h_{t}^{*}}=\exp \left\{\frac{1}{\gamma} \int_{0}^{t}\left(\widehat{r}_{s}+\frac{1}{2} \lambda^{2}-\delta\right) \mathrm{d} s+\frac{\bar{y}}{\gamma}\right\} \exp \left\{\frac{\lambda}{\gamma} \int_{0}^{t} \mathrm{~d} W_{s}\right\} .
$$

Here, $\bar{y}=-\left(\log y+\log \widehat{M}_{0}\right)$. 
We can write the habit level $h_{t}^{*}$ as follows:

$$
\begin{aligned}
h_{t}^{*}= & \exp \left\{\int_{0}^{t} \beta \exp \{-(\alpha-\beta)(t-s)\} \log \widehat{c}_{s}^{*} \mathrm{~d} s\right\} \\
= & \exp \left\{\int_{0}^{t} \beta \exp \{-(\alpha-\beta)(t-s)\}\right. \\
& \left.\times\left[\frac{1}{\gamma} \int_{0}^{s}\left(\widehat{r}_{u}+\frac{1}{2} \lambda^{2}-\delta\right) \mathrm{d} u+\frac{\bar{y}}{\gamma}+\frac{\lambda}{\gamma} \int_{0}^{s} \mathrm{~d} W_{u}\right] \mathrm{d} s\right\} \\
= & \exp \left\{\int_{0}^{t}\left(\frac{1}{\gamma} Q_{t-s}-\frac{1}{\gamma}\right)\left(\widehat{r}_{s}+\frac{1}{2} \lambda^{2}-\delta\right) \mathrm{d} s\right\} \\
& \times \exp \left\{\left(\frac{1}{\gamma} Q_{t}-\frac{1}{\gamma}\right) \bar{y}+\int_{0}^{t}\left(\frac{\lambda}{\gamma} Q_{t-s}-\frac{\lambda}{\gamma}\right) \mathrm{d} W_{s}\right\} .
\end{aligned}
$$

Here, $Q_{t-s}=1+\frac{\beta}{\alpha-\beta}[1-\exp \{-(\alpha-\beta)(t-s)\}]$.

Hence,

$$
\begin{aligned}
c_{t}^{*} & =h_{t}^{*} \exp \left\{\frac{1}{\gamma} \int_{0}^{t}\left(\widehat{r}_{s}+\frac{1}{2} \lambda^{2}-\delta\right) \mathrm{d} s+\frac{\bar{y}}{\gamma}\right\} \exp \left\{\frac{\lambda}{\gamma} \int_{0}^{t} \mathrm{~d} W_{s}\right\} \\
& =\left(c_{0}^{*}\right)^{Q_{t}} \exp \left\{\frac{1}{\gamma} \int_{0}^{t} Q_{t-s}\left(\widehat{r}_{s}+\frac{1}{2} \lambda^{2}-\delta\right) \mathrm{d} s+\frac{\lambda}{\gamma} \int_{0}^{t} Q_{t-s} \mathrm{~d} W_{s}\right\} .
\end{aligned}
$$

It follows from $(52)$ that $q_{t-s}=\lambda Q_{t-s} /(\gamma \sigma)$ models the sensitivity of log consumption $\log c_{t}^{*}$ to the stock return shock $\sigma \mathrm{d} W_{s}$.

Subtracting $\log c_{t+\tau}^{*}$ from $\log c_{t}^{*}$ and taking the limit $\tau \rightarrow 0$, we arrive at (26).

\section{A.3 Proof of Theorem 4.2}

Straightforward computations show that

$$
\begin{aligned}
V_{t, \tau}= & \mathbb{E}_{t}\left[\frac{M_{t+\tau}}{M_{t}} c_{t+\tau}^{*}\right] \\
= & c_{t}^{*} G_{t, \tau} \mathbb{E}_{t}\left[\exp \left\{-\int_{0}^{\tau}\left(r+\frac{1}{2} \lambda^{2}\right) \mathrm{d} v-\lambda \int_{0}^{\tau} \mathrm{d} W_{t+\tau-v}\right\}\right. \\
& \left.\times \exp \left\{\frac{1}{\gamma} \int_{0}^{\tau} Q_{v}\left(\widehat{r}_{t+\tau-v}+\frac{1}{2} \lambda^{2}-\delta\right) \mathrm{d} v+\frac{\lambda}{\gamma} \int_{0}^{\tau} Q_{v} \mathrm{~d} W_{t+\tau-v}\right\}\right] \\
= & c_{t}^{*} G_{t, \tau} C_{t, \tau}
\end{aligned}
$$


where

$$
\begin{aligned}
& G_{t, \tau}=\left(c_{0}^{*}\right)^{\left(Q_{t+\tau}-Q_{t}\right)} \exp \left\{\frac{1}{\gamma} \int_{0}^{t}\left(Q_{t+\tau-s}-Q_{t-s}\right)\left(\widehat{r}_{s}+\frac{1}{2} \lambda^{2}-\delta\right) \mathrm{d} s\right\} \\
& \times \exp \left\{\frac{\lambda}{\gamma} \int_{0}^{t}\left(Q_{t+\tau-s}-Q_{t-s}\right) \mathrm{d} W_{s}\right\}, \\
& C_{t, \tau}=\exp \left\{-\int_{0}^{\tau}\left(r-Q_{v} \frac{1}{\gamma}\left[\widehat{r}_{t+\tau-v}+\frac{1}{2} \lambda^{2}-\delta\right]+Q_{v} \frac{\lambda^{2}}{\gamma}-\frac{1}{2} Q_{v}^{2} \frac{\lambda^{2}}{\gamma^{2}}\right) \mathrm{d} v\right\} .
\end{aligned}
$$

Eqn. (53) shows that the term $V_{t, \tau} / c_{t}^{*}$ consists of two factors. The factor $G_{t, \tau}$ represents past stock return shocks that the individual absorbs into future growth rates of (median) consumption. This factor equals unity if the individual directly absorbs unexpected stock returns shocks into current consumption. The factor $C_{t, \tau}$ summarizes the impacts of the unconditional growth rates of median consumption and the future (uncertain) rates of return on the market value of future consumption.

It follows from Itô's lemma that $\log V_{t}=\log \left[\int_{0}^{T-t} V_{t, \tau} \mathrm{d} \tau\right]$ satisfies

$$
\mathrm{d} \log V_{t}=(\ldots) \mathrm{d} t+\frac{\lambda}{\gamma} \int_{0}^{T-t} Q_{\tau} \frac{V_{t, \tau}}{V_{t}} \mathrm{~d} \tau \cdot \mathrm{d} W_{t}
$$

suppressing the drift term for brevity. It also holds that (this follows from applying Itô's lemma to the dynamic budget constraint (7))

$$
\mathrm{d} \log A_{t}=(\ldots) \mathrm{d} t+\sigma \cdot \frac{\pi_{t}}{A_{t}} \cdot \mathrm{d} W_{t}
$$

Setting Eqn. (57) equal to Eqn. (56) and solving for the approximate optimal portfolio choice, we arrive at 28 .

\section{A.4 Proof of Theorem 5.1}

We first write the individual's consumption choice $c_{t}^{*}$ in terms of unexpected past stock return and interest rate shocks. The stochastic discount factor $\widehat{M}_{t} \equiv M_{t}\left(1+\beta P_{t}\right)$ 
is given by

$$
\widehat{M}_{t}=\widehat{M}_{0} \exp \left\{-\int_{0}^{t}\left(\widehat{r}_{s}+\frac{1}{2}\left\|\widehat{\lambda}_{s}\right\|^{2}\right) \mathrm{d} s\right\} \exp \left\{-\widehat{\lambda}_{s}^{\top} \int_{0}^{t} \mathrm{~d} W_{s}\right\}
$$

where

$$
\widehat{r}_{s}=\beta+\frac{r_{s}-\alpha \beta P_{s}}{1+\beta P_{s}}, \quad \widehat{\lambda}_{1, s}=\lambda_{1}+\beta \frac{\sigma_{r} \rho \widehat{D}_{s} P_{s}}{1+\beta P_{s}}, \quad \widehat{\lambda}_{2, s}=\lambda_{2}+\beta \frac{\sigma_{r} \sqrt{1-\rho^{2}} \widehat{D}_{s} P_{s}}{1+\beta P_{s}}
$$

with

$$
\widehat{D}_{s}=\int_{0}^{T-s} \alpha_{s, \tau} D_{\tau} \mathrm{d} \tau
$$

Here,

$$
\begin{gathered}
D_{\tau}=\frac{1-\exp \{-\kappa \tau\}}{\kappa}, \\
\alpha_{s, \tau}=\frac{e^{-\int_{0}^{\tau}\left(\alpha-\beta+r_{s}+\kappa D_{u}\left(\bar{r}-r_{s}\right)-\sigma_{r} D_{u}\left(\lambda_{1} \rho+\lambda_{2} \sqrt{1-\rho^{2}}\right)-\frac{1}{2} \sigma_{r}^{2} D_{u}^{2}\right) \mathrm{d} u}}{\int_{0}^{T-s} e^{-\int_{0}^{\tau}\left(\alpha-\beta+r_{s}+\kappa D_{u}\left(\bar{r}-r_{s}\right)-\sigma_{r} D_{u}\left(\lambda_{1} \rho+\lambda_{2} \sqrt{1-\rho^{2}}\right)-\frac{1}{2} \sigma_{r}^{2} D_{u}^{2}\right) \mathrm{d} u} \mathrm{~d} \tau} .
\end{gathered}
$$

Substituting (58) into (21), we arrive at

$$
\widehat{c}_{t}^{*}=\frac{c_{t}^{*}}{h_{t}^{*}}=\exp \left\{\frac{1}{\gamma} \int_{0}^{t}\left(\widehat{r}_{s}+\frac{1}{2}\left\|\widehat{\lambda}_{s}\right\|^{2}-\delta\right) \mathrm{d} s+\frac{\bar{y}}{\gamma}\right\} \exp \left\{\frac{1}{\gamma} \widehat{\lambda}_{s}^{\top} \int_{0}^{t} \mathrm{~d} W_{s}\right\} .
$$

Here, $\bar{y}=-\left(\log y+\log \widehat{M}_{0}\right)$.

We find (see the derivation of (51) and (52))

$$
c_{t}^{*}=\left(c_{0}^{*}\right)^{Q_{t}} \exp \left\{\frac{1}{\gamma} \int_{0}^{t} Q_{t-s}\left(\widehat{r}_{s}+\frac{1}{2}\left\|\widehat{\lambda}_{s}\right\|^{2}-\delta\right) \mathrm{d} s+\frac{1}{\gamma} \int_{0}^{t} Q_{t-s} \widehat{\lambda}_{s}^{\top} \mathrm{d} W_{s}\right\} .
$$

The market value at time $t$ of the future consumption stream $\left\{c_{s}^{*}\right\}_{t \leq s \leq T}$, i.e., $V_{t}=\int_{0}^{T-t} V_{t, \tau} \mathrm{d} \tau$, is a function of the state variables $r_{t}$ and $\log \widehat{M}_{t}$. It now follows from 
Itô's lemma that

$$
\begin{aligned}
\mathrm{d} \log V_{t} & =(\ldots) \mathrm{d} t-\left(\widehat{\lambda}_{1, t} \frac{\partial V_{t}}{\partial \log \widehat{M}_{t}} \frac{1}{V_{t}}-\sigma_{r} \rho \frac{\partial V_{t}}{\partial r_{t}} \frac{1}{V_{t}}\right) \mathrm{d} W_{1, t} \\
& -\left(\widehat{\lambda}_{2, t} \frac{\partial V_{t}}{\partial \log \widehat{M}_{t}} \frac{1}{V_{t}}-\sigma_{r} \sqrt{1-\rho^{2}} \frac{\partial V_{t}}{\partial r_{t}} \frac{1}{V_{t}}\right) \mathrm{d} W_{2, t} .
\end{aligned}
$$

It also holds that

$$
\mathrm{d} \log A_{t}=(\ldots) \mathrm{d} t+\left(\frac{\pi_{1, t}}{A_{t}} \sigma_{S}-\frac{\pi_{2, t}}{A_{t}} \sigma_{r} \rho D_{T_{1}-t}\right) \mathrm{d} W_{1, t}-\frac{\pi_{2, t}}{A_{t}} \sigma_{r} \sqrt{1-\rho^{2}} D_{T_{1}-t} \mathrm{~d} W_{2, t} .
$$

Setting Eqn. (66) equal to Eqn. (65) and solving for the approximate optimal portfolio choice, we arrive at (33) and (34).

\section{A.5 Proof of Theorem 6.1}

Given $\widehat{A}_{0}$, the approximate optimal relative consumption choice $\widehat{c}_{t}^{*}$ can be obtained from Schroder and Skiadas (1999). Finally, the approximate optimal consumption choice $c_{t}^{*}$ follows as in Eqn. (15).

\section{B Excessive Median Growth Rates of Consumption}

We state the following theorem.

Theorem B.1. Suppose that $r_{t}$ does not depend on time (i.e., $r_{t}=r$ ) and let $\widehat{r}_{t}=$ $\beta+\left(r-\alpha \beta P_{t}\right) /\left(1+\beta P_{t}\right)$. Then:

1. The value of $\widehat{r}_{t}$ increases as the preference parameter $\beta$ increases, given fixed $\alpha-\beta$.

2. The value of $\widehat{r}_{t}$ decreases as $T$ increases. In particular, $\widehat{r}_{t} \rightarrow r$ if $T \rightarrow \infty$.

Theorem B.1 and the decomposition in 26) imply that current consumption has a large impact on future habit levels if the preference parameter $\beta$ is large. Also, the utility gain of an increase in consumption is smaller when the agent is (relatively) young (i.e., small $t$ ) than when the agent is (relatively) old (i.e., large $t$ ). Hence, an agent with habit 
preferences prefers high unconditional median growth rates of log consumption (especially at high ages) except when his subjective time preference rate $\delta$ is excessive.

\section{B.1 Proof of Theorem B.1}

We first prove that the (partial) derivative of $\widehat{r}_{t}$ with respect to $\beta$ is positive given fixed $\alpha-\beta$. Define $\eta=\alpha-\beta$. Substituting $\alpha=\eta+\beta$ into (B.1), we find

$$
\widehat{r}_{t}=\beta+\frac{r-(\eta+\beta) \beta P_{t}}{1+\beta P_{t}}
$$

The (partial) derivative of $\widehat{r}_{t}$ with respect to $\beta$ is given by

$$
\begin{aligned}
\frac{\partial \widehat{r}_{t}}{\partial \beta} & =1+\frac{-\left(1+\beta P_{t}\right)(\eta+2 \beta) P_{t}-\left(r-(\eta+\beta) \beta P_{t}\right) P_{t}}{\left(1+\beta P_{t}\right)^{2}} \\
& =1+\frac{-\eta P_{t}-2 \beta P_{t}-\left(\beta P_{t}\right)^{2}-r P_{t}}{1+2 \beta P_{t}+\left(\beta P_{t}\right)^{2}}
\end{aligned}
$$

Hence,

$$
\begin{aligned}
\frac{\partial \widehat{r}_{t}}{\partial \beta} \geq 0 & \Leftrightarrow \frac{-\eta P_{t}-2 \beta P_{t}-\left(\beta P_{t}\right)^{2}-r P_{t}}{1+2 \beta P_{t}+\left(\beta P_{t}\right)^{2}} \geq-1 \\
& \Leftrightarrow(r+\eta) P_{t} \leq 1 \Leftrightarrow 1-\exp \{-(r+\eta)(T-t)\} \leq 1 .
\end{aligned}
$$

Hence, $\partial \widehat{r}_{t} / \partial \beta$ is positive given fixed $\alpha-\beta$.

Finally, we prove that the (partial) derivative of $\widehat{r}_{t}$ with respect to $T$ is negative. The (partial) derivative of $\widehat{r}_{t}$ with respect to $T$ is given by

$$
\frac{\partial \widehat{r}_{t}}{\partial T}=-r\left(1+\beta P_{t}\right)^{-2} \frac{\partial P_{t}}{\partial T}-\alpha \beta\left(1+\beta P_{t}\right)^{-2} \frac{\partial P_{t}}{\partial T}
$$

Using the fact that $\partial P_{t} / \partial T>0$, we find that $\partial \widehat{r}_{t} / \partial T<0$. Furthermore, simple algebra yields that $\widehat{r}_{t}=r$ if $T=\infty$. Here, we use the fact that $P_{t} \rightarrow 1 /(r+\alpha-\beta)$ as $T \rightarrow \infty$. 


\section{Numerical Solution Method}

To assess the accuracy of our pathwise approximation, we also determine the genuine optimal consumption and portfolio policies using numerical backward induction. Because we only explore the case $\alpha=\beta$, we can reduce the number of state variables from two (i.e., wealth level and habit level) to one (i.e., wealth-to-habit ratio). The first step is to specify discrete points in the state space, called grid points. For each grid point, we determine the optimal relative consumption choice and the optimal portfolio choice. To determine the optimal policies, we need to evaluate the utility value for every combination of relative consumption choice and portfolio choice. The utility value is equal to the sum of current utility and the discounted expected continuation value. Once we have computed the utility value for every combination of relative consumption choice and portfolio choice, we select the maximum utility value. We then use this maximum utility value to solve the previous period's maximization problem. This process is iterated backwards in time until the entire problem has been solved. In the last period, the optimal relative consumption

choice and the maximum utility value are given by $\widehat{c}_{T}^{\mathrm{opt}}=A_{T} / h_{T}$ and $\left(\widehat{c}_{T}^{\mathrm{opt}}\right)^{1-\gamma} /(1-\gamma)$, respectively. This gives us the terminal condition for the backward induction procedure. We use Gaussian quadrature to compute expectations. For points that do not lie on the state space grid, we evaluate the utility level using cubic spline interpolation.

We introduce the following notation:

- $\mathcal{S}$ : total number of simulations;

- $\Delta t$ : time step;

- $t_{n}=n \Delta t$ for $n=0, \ldots,\left\lfloor\frac{T}{\Delta t}\right\rfloor$.

The floor operator $\lfloor\cdot\rfloor$ rounds a number downward to its nearest integer.

We now apply the following steps:

1. We generate $\mathcal{S}$ trajectories of the stochastic discount factor $(s=1, \ldots, \mathcal{S})$ :

$$
M_{s, t_{n+1}}=M_{s, t_{n}}-r M_{s, t_{n}} \Delta t-\lambda M_{s, t_{n}} \sqrt{\Delta t} \epsilon_{s, t_{n}}, \quad n=0, \ldots,\left\lfloor\frac{T}{\Delta t}\right\rfloor .
$$

Here, $\epsilon_{s, t_{n}}$ is a standard normally distributed random variable. 
2. We compute the approximate relative consumption choice $\widehat{c}_{s, t_{n}}^{*}$ and the approximate portfolio strategy $\pi_{s, t_{n}}^{*}$ for $s=1, \ldots, \mathcal{S}$ and $n=0, \ldots,\left\lfloor\frac{T}{\Delta t}\right\rfloor$. We note that the approximate relative consumption choice $\widehat{c}_{s, t_{n}}^{*}$ is a function of the stochastic discount factor $\widehat{M}_{s, t_{n}} \equiv M_{s, t_{n}}\left(1+\beta P_{t_{n}}\right)$. We obtain the individual's lifetime utility $U(c / h)$ by using the method of numerical backward induction. Note that in this step we do not use backward induction to obtain the optimal solutions.

3. We numerically solve for the certainty equivalent consumption $c e^{*}$.

4. We compute the optimal consumption strategy $c_{s, t_{n}}^{\mathrm{opt}}$ and the optimal portfolio strategy $\pi_{s, t_{n}}^{\mathrm{opt}}$ for $s=1, \ldots, \mathcal{S}$ and $n=0, \ldots,\left\lfloor\frac{T}{\Delta t}\right\rfloor$. Lifetime utility follows from the backward induction algorithm.

5. We numerically solve for the optimal certainty equivalent consumption $c e^{\text {opt }}$.

6. Finally, we compute the welfare loss $l=\left(c e^{\mathrm{opt}}-c e^{*}\right) / c e^{\mathrm{opt}}$.

\section{REFERENCES}

Abel, A., 1999. Risk premia and term premia in general equilibrium. Journal of Monetary Economics $43,3-33$.

Abel, A. B., 1990. Asset prices under habit formation and catching up with the Joneses. American Economic Review 80, 38-42.

Ang, A., Bekaert, G., Lui, J., 2005. Why stocks may disappoint. Journal of Financial Economics 76, 471-508.

Bell, D. E., 1982. Regret in decision making under uncertainty. Operations Research 30, 961-981.

Bell, D. E., 1983. Risk premiums for decision regret. Management Science 29, 1156-1166.

Bell, D. E., 1985. Disappointment in decision making under uncertainty. Operations Research 33, 1-27.

Berkelaar, A. B., Kouwenberg, R., Post, T., 2004. Optimal portfolio choice under loss aversion. Review of Economics and Statistics 86, 973-987.

Bhamra, H. S., Uppal, R., 2006. The role of risk aversion and intertemporal substitution in dynamic consumption-portfolio choice with recursive utility. Journal of Economic Dynamics and Control 30, 967-991.

Van Bilsen, S., Laeven, R. J. A., Nijman, Th.. E., 2017. Consumption and portfolio choice under loss aversion and endogenous updating of the reference level, Working Paper.

Bodie, Z., Detemple, J. B., Otruba, S., Walter, S., 2004. Optimal consumption-portfolio choices and retirement planning. Journal of Economic Dynamics and Control 28, 1115-1148.

Bodie, Z., Merton, R. C., Samuelson, W. F., 1992. Labor supply flexibility and portfolio choice in a life-cycle model. Journal of Economic Dynamics and Control 16, 427-449.

Bowman, D., Minehart, D., Rabin, M., 1999. Loss aversion in a consumption-savings model. Journal of Economic Behavior and Organization 38, 155-178.

Brennan, M. J., Xia, Y., 2002. Dynamic asset allocation under inflation. Journal of Finance 57, 12011238.

Campbell, J. Y., Cocco, J., Gomes, F., Maenhout, P. J., Viceira, L. M., 2001. Stock market mean reversion and the optimal equity allocation of a long-lived investor. European Finance Review 5, 269-292. 
Campbell, J. Y., Cochrane, J., 1999. By force of habit: A consumption-based explanation of aggregate stock market behavior. Journal of Political Economy 107, 205-251.

Campbell, J. Y., Deaton, A., 1989. Why is consumption so smooth? Review of Economic Studies 56, 357-373.

Campbell, J. Y., Mankiw, N. G., 1991. The response of consumption to income: A cross-country investigation. European Economic Review 35, 723-767.

Campbell, J. Y., Viceira, L. M., 1999. Consumption and portfolio decisions when expected returns are time varying. The Quarterly Journal of Economics 114, 433-495.

Carroll, C. D., 2000. Solving consumption models with multiplicative habits. Economics Letters 68, $67-77$.

Carroll, C. D., Overland, J., Weil, D. N., 1997. Comparison utility in a growth model. Journal of Economic Growth 2, 339-367.

Carroll, C. D., Overland, J., Weil, D. N., 2000. Saving and growth with habit formation. American Economic Review 90, 341-355.

Chacko, G., Viceira, L. M., 2005. Dynamic consumption and portfolio choice with stochastic volatility in incomplete markets. Review of Financial Studies 18, 1369-1402.

Chan, Y. L., Kogan, L., 2002. Catching up with the Joneses: Heterogeneous preferences and the dynamics of asset prices. Journal of Political Economy 110, 1255-1285.

Chapman, D. A., 1998. Habit formation and aggregate consumption. Econometrica 66, 1223-1230.

Cocco, J. F., 2005. Portfolio choice in the presence of housing. Review of Financial Studies 18, 535-567.

Cocco, J. F., Gomes, F. J., Maenhout, P. J., 2005. Consumption and portfolio choice over the life cycle. Review of Financial Studies 18, 491-533.

Constantinides, G. M., 1990. Habit formation: A resolution of the equity premium puzzle. Journal of Political Economy 98, 519-543.

Corrado, L., Holly, S., 2011. Multiplicative habit formation and consumption: A note. Economics Letters $113,116-119$.

Cox, J. C., Huang, C., 1989. Optimal consumption and portfolio policies when asset prices follow a diffusion process. Journal of Economic Theory 49, 33-83.

Cox, J. C., Huang, C., 1991. A variational problem arising in financial economics. Journal of Mathematical Economics 20, 465-487.

Crawford, I., 2010. Habits revealed. Review of Economic Studies 77, 1382-1402.

Crossley, T. F., Low, H., O'Dea, C., 2013. Household consumption through recent recessions. Journal of Applied Public Economics 34, 203-229.

Deaton, A., 1987. Life-cycle models of consumption: Is the evidence consistent with the theory? In: Bewley, T. F. (ed.), Advances in Econometrics: Fifth World Congress, Cambridge University Press, vol. 2, pp. 121-148.

Deaton, A., 1992. Understanding Consumption. Oxford University Press.

Detemple, J. B., Zapatero, F., 1991. Asset prices in an exchange economy with habit formation. Econometrica 59, 1633-1657.

Detemple, J. B., Zapatero, F., 1992. Optimal consumption-portfolio policies with habit formation. Mathematical Finance 2, 251-274.

Duffie, D., Epstein, L. G., 1992. Stochastic differential utility. Econometrica 60, 353-394.

Duffie, D., Skiadas, C., 1992. Continuous-time security pricing: A utility gradient approach. Journal of Mathematical Economics 23, 107-131.

Dus, I., Maurer, R., Mitchell, O. S., 2005. Betting on death and capital markets in retirement: A shortfall risk analysis of life annuities. Financial Services Review 14, 169-196.

Edwards, R. D., 2008. Health risk and portfolio choice. Journal of Business and Economics Statistics $26,472-485$.

Epstein, L. G., Zin, S. E., 1989. Substitution, risk aversion, and the temporal behavior of consumption and asset returns: A theoretical framework. Econometrica 57, 937-969.

Flavin, M., 1985. Excess sensitivity of consumption to current income: Liquidity constraints or myopia? Canadian Journal of Economics 18, 117-136. 
Fuhrer, J. C., 2000. Habit formation in consumption and its implications for monetary-policy models. American Economic Review 90, 367-390.

Gomes, F., Michaelides, A., 2003. Portfolio choice with internal habit formation: A life-cycle model with uninsurable labor income risk. Review of Economic Dynamics 6, 729-766.

Gomes, F., Michaelides, A., 2005. Optimal life-cycle asset allocation: Understanding the empirical evidence. Journal of Finance 60, 869-904.

Gomes, F. J., Kotlikoff, L. J., Viceira, L. M., 2008. Optimal life-cycle investing with flexible labor supply: A welfare analysis of life-cycle funds. American Economic Review 98, 297-303.

Gómez, J.-P., Priestley, R., Zapatero, F., 2009. Implications of keeping-up-with-the-joneses behavior for the equilibrium cross section of stock returns: International evidence. Journal of Finance 64, 2703-2737.

Guasoni, P., Huberman, G., Ren, D., 2015. Shortfall aversion, Working Paper.

Guillén, M., Jørgensen, P. L., Nielsen, J. P., 2006. Return smoothing mechanisms in life and pension insurance: Path-dependent contingent claims. Insurance: Mathematics and Economics 38, 229-252.

Guillén, M., Nielsen, J. P., Pérez-Marín, A. M., Petersen, K. S., 2013. Performance measurement of pension strategies: A case study of Danish life-cycle products. Scandinavian Actuarial Journal 2013, 49-68.

Gul, F., 1991. A theory of disappointment aversion. Econometrica 59, 667-686.

Horneff, W. J., Maurer, R. H., Mitchell, O. S., Dus, I., 2008. Following the rules: Integrating asset allocation and annuitization in retirement portfolios. Insurance: Mathematics and Economics 42, 396-408.

Jørgensen, P. L., Linnemann, P., 2012. A comparison of three different pension savings products with special emphasis on the payout phase. Annals of Actuarial Science 6, 137-152.

Kahneman, D., Tversky, A., 1979. Prospect theory: An analysis of decision under risk. Econometrica 47, 263-292.

Karatzas, I., Lehoczky, J. P., Shreve, S. E., 1987. Optimal consumption and portfolio decisions for a "small investor" on a finite horizon. SIAM Journal of Control and Optimization 25, 1557-1586.

Karatzas, I., Shreve, S. E., 1998. Methods of Mathematical Finance, vol. 39. Springer.

Köszegi, B., Rabin, M., 2006. A model of reference-dependent preferences. Quarterly Journal of Economics 121, 1133-1165.

Köszegi, B., Rabin, M., 2007. Reference-dependent risk attitudes. American Economic Review 97, 10471073.

Köszegi, B., Rabin, M., 2009. Reference-dependent consumption plans. American Economic Review 99, 909-936.

Kozicki, S., Tinsley, P. A., 2002. Dynamic specifications in optimizing trend-deviation macro models. Journal of Economic Dynamics and Control 26, 1585-1611.

Kraft, H., Seifried, F. T., 2014. Stochastic differential utility as the continuous-time limit of recursive utility. Journal of Economic Theory 151, 528-550.

Kreps, D. M., Porteus, E. L., 1978. Temporal resolution of uncertainty and dynamic choice theory. Econometrica 46, 185-200.

Laeven, R. J. A., Stadje, M. A., 2014. Robust portfolio choice and indifference valuation. Mathematics of Operations Research 39, 1109-1141.

Linnemann, P., Bruhn, K., Steffensen, M., 2014. A comparison of modern investment-linked pension savings products. Annals of Actuarial Science 9, 72-84.

Liu, J., 2007. Portfolio selection in stochastic environments. Review of Financial Studies 20, 1-39.

Loomes, G., Sugden, R., 1982. Regret theory: An alternative theory of rational choice under uncertainty. Economic Journal 92, 805-824.

Loomes, G., Sugden, R., 1986. Disappointment and dynamic consistency in choice under uncertainty. Review of Economic Studies 53, 271-282.

Maurer, R., Mitchell, O. S., Rogalla, R., Siegelin, I., 2016. Accounting and actuarial smoothing of retirement payouts in participating life annuities. Insurance: Mathematics and Economics 71, 268283. 
Maurer, R., Rogalla, R., Siegelin, I., 2013a. Participating payout annuities: Lessons from Germany. ASTIN Bulletin 43, 159-187.

Maurer, R. H., Mitchell, O. S., Rogalla, R., Kartashov, V., 2013b. Life cycle portfolio choice with systematic longevity risk and variable investment-linked deferred annuities. Journal of Risk and Insurance 80, 649-676.

Merton, R. C., 1969. Lifetime portfolio selection under uncertainty: The continuous-time case. Review of Economics and Statistics 51, 247-257.

Merton, R. C., 1971. Optimum consumption and portfolio rules in a continuous-time model. Journal of Economic Theory 3, 373-413.

Merton, R. C., 2014. The crisis in retirement planning, Harvard Business Review.

Morningstar, 2017. 2017 Target-Date Fund Landscape. Answers to Frequently Asked Questions.

Mossin, J., 1968. Optimal multiperiod portfolio policies. Journal of Business 41, 215-229.

Muermann, A., Mitchell, O. S., Volkman, J. M., 2006. Regret, portfolio choice, and guarantees in defined contribution schemes. Insurance: Mathematics and Economics 39, 219-229.

Munk, C., 2008. Portfolio and consumption choice with stochastic investment opportunities and habit formation in preferences. Journal of Economic Dynamics and Control 32, 3560-3589.

Pagel, M., 2017. Expectations-based reference-dependent life-cycle consumption. Review of Economic Studies 84, 885-934.

Pliska, S. R., 1986. A stochastic calculus model of continuous trading: Optimal portfolios. Mathematics of Operations Research 11, 371-382.

Quiggin, J., 1994. Regret theory with general choice sets. Journal of Risk and Uncertainty 8, 153-165.

Samuelson, P. A., 1969. Lifetime portfolio selection by dynamic stochastic programming. Review of Economics and Statistics 51, 239-246.

Samwick, A. A., 1998. Discount rate heterogeneity and social security reform. Journal of Development Economics 57, 117-146.

Schroder, M., Skiadas, C., 1999. Optimal consumption and portfolio selection with stochastic differential utility. Journal of Economic Theory 89, 68-126.

Schroder, M., Skiadas, C., 2002. An isomorphism between asset pricing models with and without linear habit formation. Review of Financial Studies 15, 1189-1221.

Smith, W. T., Zhang, Q., 2007. Asset pricing with multiplicative habit and power-expo preferences. Economics Letters 94, 319-325.

Sugden, R., 1993. An axiomatic foundation for regret theory. Journal of Economic Theory 60, 159-180.

Sundaresan, S. M., 1989. Intertemporally dependent preferences and the volatility of consumption and wealth. Review of Financial Studies 2, 73-89.

Tversky, A., Kahneman, D., 1992. Advances in prospect theory: Cumulative representation of uncertainty. Journal of Risk and Uncertainty 5, 297-323.

Viceira, L. M., 2001. Optimal portfolio choice for long-horizon investors with nontradable labor income. Journal of Finance 56, 433-470.

Wachter, J. A., 2002. Portfolio and consumption decisions under mean-reverting returns: An exact solution for complete markets. Journal of Financial and Quantitative Analysis 37, 63-91.

Yao, R., Zhang, H. H., 2005. Optimal consumption and portfolio choices with risky housing and borrowing constraints. Review of Financial Studies 18, 197-239. 